CONFORMAL GEOMETRY AND DYNAMICS

An Electronic Journal of the American Mathematical Society

Volume 9, Pages 1-23 (February 9, 2005)

S $1088-4173(05) 00118-9$

\title{
CONVERGENCE IN DISCRETE CAUCHY PROBLEMS AND APPLICATIONS TO CIRCLE PATTERNS
}

\author{
D. MATTHES
}

\begin{abstract}
A lattice-discretization of analytic Cauchy problems in two dimensions is presented. It is proven that the discrete solutions converge to a smooth solution of the original problem as the mesh size $\varepsilon$ tends to zero. The convergence is in $C^{\infty}$ and the approximation error for arbitrary derivatives is quadratic in $\varepsilon$. In application, $C^{\infty}$-approximation of conformal maps by Schramm's orthogonal circle patterns and lattices of cross-ratio minus one is shown.
\end{abstract}

\section{INTRODUCTION}

In the flourishing field of discrete differential geometry, classical geometrical objects are matched by discrete counterparts which inherit many qualitative features of the smooth originals. Discretizations have been proposed for a large variety of surfaces, coordinate systems and maps; see [BP2] for an overview.

Special attention has been devoted to circle packings in the plane and their relation to conformal mappings. The fundamental question of quantitative approximation has been intensively studied. As a key result in this context, Thurston's conjecture on the convergence of hexagonal circle packings to the Riemann map was proven by Rodin and Sullivan [RS] in 1987. Their result has been improved in many ways. For instance, hexagonal packings were shown to converge in $C^{\infty}[\mathrm{HS}$, and the error for the approximation and its derivatives was estimated [DHR].

An alternative approach to a discrete theory of conformal maps is provided by circle patterns. Generally, a circle pattern with square-grid combinatorics is a correspondence that assigns to any vertex of the $\mathbb{Z}^{2}$-lattice a circle in the complex plane. As the collection of image circles inherits the square-grid combinatorics, there is a natural notion of neighbors and elementary quadruples (circles assigned to the corners of a $\mathbb{Z}^{2}$-square). One requires that the circles of an elementary quadruple intersect in one point. Thus, each circle has four points of intersection with its neighbors. The intersection points form a lattice with square-grid combinatorics on their own.

Additional conditions are imposed to single out subclasses of more rigid patterns. The most prominent subclass is provided by "orthogonal circle patterns" introduced by Schramm [Sch], also called Schramm-patterns in the following. The additional constraint is that neighboring circles intersect orthogonally.

Received by the editors March 19, 2004 and, in revised form, November 16, 2004.

2000 Mathematics Subject Classification. Primary 30G25; Secondary 35A10, 52C15.

Supported by the SFB 288 "Differential Geometry and Quantum Physics" of the Deutsche Forschungsgemeinschaft.

(C)2005 American Mathematical Society Reverts to public domain 28 years from publication 
As a second possibility, one requires that the four points of intersection on each circle have cross ratio minus one. In this situation, it is preferred to consider the lattice of intersection points rather than the circles themselves. One obtains crossratio-lattices or CR-mappings, which were first investigated by Nijhoff et al [NQC]. Generalizing CR-mappings to immersions in three-space, a definition of discrete isothermic surfaces was obtained by Bobenko and Pinkall [BP1].

In comparison to (especially hexagonal) packings, much less in known about the approximation properties of circle patterns. The $C^{0}$-convergence theorem in $\mathrm{Sch}$ seems to be the only result in this direction so far. In this article, it is proven that an arbitrary planar conformal map $u: \Omega \subset \mathbb{C} \rightarrow \mathbb{C}$ can be locally approximated by a sequence of Schramm-patterns and CR-mappings. More precisely: intersect the domain $\Omega$ with a square grid of mesh size $\varepsilon>0$, obtaining a discrete set $\Omega^{\varepsilon}$. A suitable circle pattern from the respective class can be defined on each $\Omega^{\varepsilon}$, so that the circle centers (Schramm-pattern) or intersection points (CR-mapping) approximate the values of $u$ at corresponding sites with an error $\mathcal{O}\left(\varepsilon^{2}\right)$. Moreover, the convergence is in $C^{\infty}$. This means that arbitrary partial derivatives of $u$ are uniformly approximated by the respective difference quotients calculated from the circle pattern, also with an error of order $\mathcal{O}\left(\varepsilon^{2}\right)$.

The analytic background for the geometric convergence result is of interest on its own. A discrete approximation theory for analytic Cauchy problems is developed in this article. Its applications are not limited to geometrical questions. Moreover, a new proof of the Cauchy-Kovalevskaya theorem - based on purely discrete constructions - is obtained as a by-product.

Consequently, the presented approach to circle patterns differs in nature from Schramm's where a boundary value problem was considered and techniques from (discrete) elliptic theory played an important role. Instead, our proof combines the following two ingredients; the first are methods which were developed for Cauchy problems associated with discrete hyperbolic equations. These have already been used to show $C^{\infty}$-convergence of discrete orthogonal coordinate systems BMS. The other ingredient is an adaptation of fundamental ideas from the proof of the (abstract) Cauchy-Kovalevskaya theorem $[\mathrm{Nag}$,, $\mathrm{Nir}$. In particular, a discrete counterpart of the scale of spaces of analytic functions is defined.

For definiteness, let us consider the Cauchy problem

$$
\begin{aligned}
\partial_{t} u(t, x) & =M \partial_{x} u(t, x)+f(u(t, x)), \\
u(0, x) & =u_{0}(x) .
\end{aligned}
$$

The continuous solution $u: \Omega \rightarrow \mathbb{C}^{d}$ is sought on $\Omega \subset \mathbb{R}^{2}$, where $M$ is a constant $d \times d$-matrix, and $f$ is an analytic function. For $x$-analytic data $u_{0}$, there exists a local solution to (1.1) by the Cauchy-Kovalevskaya theorem.

A variety of elliptic equations - e.g., the nonlinear Poisson equation $\left(\partial_{x}^{2}+\partial_{t}^{2}\right) u=$ $f(u)$ - can be brought into the standard form (1.1). In the case of most interest here, $u$ is a conformal map. Identifying the $(t, x)$-plane with the complex numbers, $u(t, x): \Omega \rightarrow \mathbb{C}$ is holomorphic in $z=x+i t$, and hence solves the Cauchy-Riemannequations, which is (1.1) with $M=i$ and $f \equiv 0$.

Circle patterns are constructed which approximate $u$. These are characterized by discrete functions $v^{\varepsilon}$ on two-dimensional grids $\Omega^{\varepsilon} \subset \Omega$ of mesh-size $\varepsilon>0$. The crucial observation is that the $v^{\varepsilon}$ solve a discrete equation similar to (1.1), 
where derivatives are replaced by difference quotients. The content of the developed approximation theory is that the discrete solutions $v^{\varepsilon}$ converge to $u$ in $C^{\infty}$. Eventually, this leads to convergence of the corresponding circle patterns.

The article is organized as follows: In section 2 the theorem about discrete approximation of the Cauchy problem (1.1) is formulated. The theorem is proven in section 3. Its numerical applicability is discussed in section 4 Convergence theorems for CR-mappings and Schramm-patterns are proven in sections 5 and 6. respectively.

Acknowledgments. The author would like to thank Alexander Bobenko and Yuri Suris for many discussions and helpful advice. Further, the author is grateful to Walter Craig for an entertaining introduction to the topic of abstract CauchyKovalevskaya theorems.

\section{Discrete approximation of the Cauchy problem}

As usual, a function $f: \mathcal{D} \subset \mathbb{C}^{p} \rightarrow \mathbb{C}^{d}$ is called analytic, if it is complex differentiable in its $p$ arguments. Moreover, a function $u: \mathrm{I}_{\xi} \rightarrow \mathbb{C}^{d}$ defined on the real interval $\mathrm{I}_{\xi}=[-\xi,+\xi]$ is called analytic if it extends to a complex differentiable function on $B_{\rho}\left(\mathrm{I}_{\xi}\right)=\left\{x \in \mathbb{C} \mid \operatorname{dist}\left(x, \mathrm{I}_{\xi}\right) \leq \rho\right\}$ for an appropriate choice of $\rho>0$. By abuse of notation, there will be no distinction between $u$ and its uniquely determined complex extension.

Consider problem (1.1) under the following assumptions: The $d \times d$-matrix $M$ is constant, $f$ is an analytic function near $u_{0}(0)$, and the initial datum $u_{0}$ is analytic on an interval $\mathrm{I}_{\xi}$. Solutions $u=\left(u_{(1)}, \ldots, u_{(d)}\right): \Omega \rightarrow \mathbb{C}^{d}$ are sought on diamondshaped domains

$$
\Omega=\Omega(r)=\left\{(t, x) \in \mathbb{R}^{2}:|x|+|t| \leq r\right\} .
$$

The Cauchy-Kovalevskaya theorem implies:

Theorem 2.1. Problem (1.1) has a classical solution $u$ on $\Omega=\Omega(r)$ for a suitable $r<\xi$. This solution is $x$-analytic and $t$-smooth, i.e., of class $C^{\infty}$.

Replace $u$ by a function $v^{\varepsilon}=\left(v_{(1)}^{\varepsilon}, \ldots, v_{(d)}^{\varepsilon}\right)$ defined on the discrete set

$$
\Omega^{\varepsilon}(r)=\{(t, x) \in \Omega(r): x+t \in \varepsilon \mathbb{Z}\},
$$

which is the intersection of $\Omega(r)$ with the 45-degree rotated standard lattice $(\lambda \mathbb{Z})^{2}$ of mesh size $\lambda=\varepsilon / \sqrt{2}$. For $v^{\varepsilon}: \Omega^{\varepsilon}(r) \rightarrow \mathbb{C}^{d}$, difference quotients

$$
\begin{aligned}
& \left(\delta_{x} v^{\varepsilon}\right)(t, x)=\frac{1}{\varepsilon}\left(v^{\varepsilon}\left(t, x+\frac{\varepsilon}{2}\right)-v^{\varepsilon}\left(t, x-\frac{\varepsilon}{2}\right)\right), \\
& \left(\delta_{t} v^{\varepsilon}\right)(t, x)=\frac{1}{\varepsilon}\left(v^{\varepsilon}\left(t+\frac{\varepsilon}{2}, x\right)-v^{\varepsilon}\left(t-\frac{\varepsilon}{2}, x\right)\right),
\end{aligned}
$$

are given on the dual lattice

$$
\Omega_{*}^{\varepsilon}(r)=\left\{(t, x) \in \Omega\left(r-\frac{\varepsilon}{2}\right): \frac{\varepsilon}{2}+x+t \in \varepsilon \mathbb{Z}\right\} .
$$

Higher difference quotients $\delta_{x}^{m} \delta_{t}^{n} v^{\varepsilon}$ are defined on

$$
\Omega_{m+n}^{\varepsilon}(r)= \begin{cases}\Omega^{\varepsilon}\left(r-(m+n) \frac{\varepsilon}{2}\right) & \text { if } m+n \text { is even, } \\ \Omega_{*}^{\varepsilon}\left(r-(m+n) \frac{\varepsilon}{2}\right) & \text { if } m+n \text { is odd. }\end{cases}
$$

Replace the Cauchy problem (1.1) by the discrete problem

$$
\begin{aligned}
\delta_{t} v^{\varepsilon}(t, x) & =M \delta_{x} v^{\varepsilon}(t, x)+f^{\varepsilon}\left(v^{\varepsilon}\right)(t, x) & & \left((t, x) \in \Omega_{*}^{\varepsilon}\right), \\
v^{\varepsilon}(0, x) & =v_{0}^{\varepsilon}(x) & & \left((0, x) \in \Omega^{\varepsilon}\right), \\
v^{\varepsilon}\left(\frac{\varepsilon}{2}, x\right) & =v_{+}^{\varepsilon}(x) & & \left(\left(\frac{\varepsilon}{2}, x\right) \in \Omega_{*}^{\varepsilon}\right) .
\end{aligned}
$$




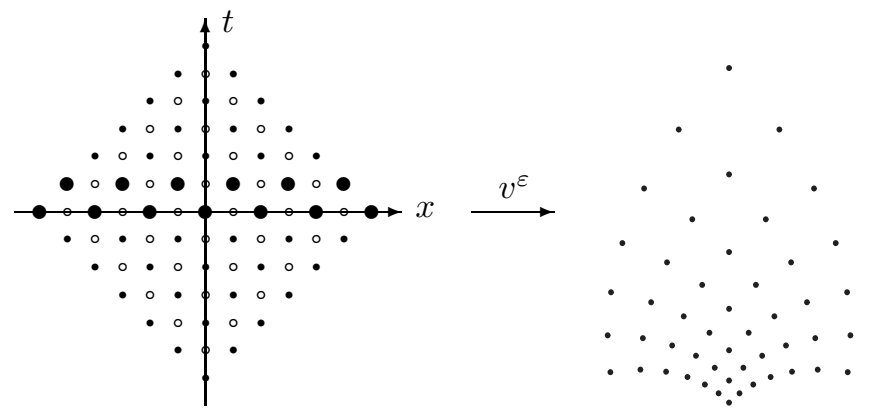

Figure 1. A CR-mapping. Points of $\Omega^{\varepsilon}(r)$ and $\Omega_{*}^{\varepsilon}$ are marked $\bullet$ and $\circ$, respectively. Initial data for $v^{\varepsilon}$ are prescribed at the bold marked sites.

For $t \gtrless 0$, the nonlinearity $f^{\varepsilon}\left(v^{\varepsilon}\right)(t, x)$ is of the form

$$
f^{\varepsilon}\left(v^{\varepsilon}\right)(t, x)=F^{\varepsilon}\left(v^{\varepsilon}\left(t, x \pm \frac{\varepsilon}{2}\right), v^{\varepsilon}\left(t, x \mp \frac{\varepsilon}{2}\right), v^{\varepsilon}\left(t \mp \frac{\varepsilon}{2}, x\right)\right) .
$$

Theorem 2.2. Assume that $u_{0}$ is analytic on $I_{\xi}$, that $f$ is analytic on a neighborhood $\mathcal{D} \subset \mathbb{C}^{d}$ of $u_{0}(0)$ and $F^{\varepsilon}$ is analytic on $\mathcal{D} \times \mathcal{D} \times \mathcal{D} \subset \mathbb{C}^{3 d}$ for each $\varepsilon>0$. Furthermore, assume that

$$
\left|F^{\varepsilon}\left(u^{+}, u^{-}, u^{*}\right)-f\left(\frac{u^{+}+u^{-}}{2}\right)\right| \leq K\left(\varepsilon+\left|u^{+}-u^{-}\right|\right)^{2}
$$

holds for all $u^{+}, u^{-}, u^{*} \in \mathcal{D}$ with $K>0$ independent of $\varepsilon$.

Then there is some $r<\xi$ such that the solutions $v^{\varepsilon}$ to problem (2.1) with

$$
\begin{aligned}
v_{0}^{\varepsilon}(x) & =u_{0}(x) & & \left((0, x) \in \Omega^{\varepsilon}(r)\right), \\
v_{+}^{\varepsilon}(x) & =u_{0}(x)+\frac{\varepsilon}{2}\left(M \partial_{x} u_{0}(x)+f\left(u_{0}(x)\right)\right) & & \left(\left(\frac{\varepsilon}{2}, x\right) \in \Omega_{*}^{\varepsilon}(r)\right)
\end{aligned}
$$

are $C^{\infty}$-convergent to a smooth function $u$ on $\Omega(r)$ with an error $\mathcal{O}\left(\varepsilon^{2}\right)$. More precisely, for arbitrary $m, n \geq 0$, there are constants $C_{m n}>0$ so that

$$
\sup _{(t, x) \in \Omega_{m+n}^{\varepsilon}(r)}\left|\partial_{x}^{m} \partial_{t}^{n} u(t, x)-\delta_{x}^{m} \delta_{t}^{n} v^{\varepsilon}(t, x)\right| \leq C_{m n} \varepsilon^{2} .
$$

The function $u$ constitutes a classical solution to the Cauchy problem (1.1).

In particular, Theorem 2.2 implies the classical existence Theorem [2.1.

Example 2.3 (A nonlinear elliptic problem). Rewrite the elliptic initial value problem,

$$
\begin{gathered}
\partial_{t}^{2} \phi(t, x)+\partial_{x}^{2} \phi(t, x)=g(\phi(x, t)), \\
\phi(0, x)=\phi_{0}(x), \partial_{t} \phi(0, x)=\phi_{+}(x)
\end{gathered}
$$

for the scalar function $\phi$ in the form of problem (1.1):

$$
\partial_{t}\left(\begin{array}{l}
u_{(1)} \\
u_{(2)} \\
u_{(3)}
\end{array}\right)=\left(\begin{array}{ccc}
0 & 0 & 0 \\
0 & 0 & 1 \\
0 & -1 & 0
\end{array}\right) \partial_{x}\left(\begin{array}{l}
u_{(1)} \\
u_{(2)} \\
u_{(3)}
\end{array}\right)+\left(\begin{array}{c}
u_{(3)} \\
0 \\
g\left(u_{(1)}\right)
\end{array}\right),
$$

denoting $u_{(1)}=\phi, u_{(2)}=\partial_{x} \phi$ and $u_{(3)}=\partial_{t} \phi$. For functions $\phi_{0}, \phi_{+}$analytic on $\mathrm{I}_{\xi}$, the respective initial data $u_{0(1)}(x)=\phi_{0}(x), u_{0(2)}=\partial_{x} \phi_{0}(x), u_{0(3)}=\phi_{+}(x)$ are analytic, too. Assuming further that the nonlinearity $g$ is analytic near $\phi_{0}(0)$, a 
solution to (2.7) exists on some $\Omega=\Omega(r)$. The constraint $\partial_{x} u_{(1)}=u_{(2)}$ propagates, so $\phi:=u_{(1)}$ indeed solves (2.5).

In order to approximate (2.7) by a discrete problem (2.1), choose

$$
F^{\varepsilon}\left(v^{+}, v^{-}, v^{*}\right)=\frac{1}{2}\left(v_{(3)}^{+}+v_{(3)}^{-}, 0, g\left(v_{(1)}^{+}\right)+g\left(v_{(1)}^{-}\right)\right)
$$

as nonlinearity, independent of $\varepsilon$. The assumptions of Theorem 2.2 concerning analyticity and boundedness of $F^{\varepsilon}$ are obviously fulfilled. A Taylor expansion of $g$ around $\bar{V}=\left(v_{(1)}^{+}+v_{(1)}^{-}\right) / 2$ proves the estimate (2.2):

$$
\frac{1}{2}\left(g\left(v_{(1)}^{+}\right)+g\left(v_{(1)}^{-}\right)\right)=g(\bar{V})+\frac{1}{2}\left(v_{(1)}^{+}-v_{(1)}^{-}\right) g^{\prime}(\bar{V})+\mathcal{O}\left(\left|v^{+}-v^{-}\right|^{2}\right) .
$$

Hence, the component $v_{(1)}^{\varepsilon}$ of the discrete solutions to problem (2.1) converges to the smooth solution $\phi$ on $\Omega(r)$ with a suitable positive $r<\xi$ in the sense of (2.4).

\section{Proof of Theorem 2.2}

The proof consists of three parts: In the first, a smooth solution $u$ to (1.1) is constructed as the limit of a suitable sequence of discrete solutions $v^{\varepsilon}$ to (2.1). Secondly, $C^{0}$-approximation of $u$ is shown, i.e., (2.4) for $m=n=0$. Finally, an induction argument yields (2.4) for arbitrary $m, n$.

The proof combines elements from discrete approximation theory for hyperbolic PDE [BMS] with ideas from Walter's proof [Wal] of the Cauchy-Kovalevskaya theorem, which is based on the classical papers [Nir, $\mathrm{Nag}$. Recall the strategy of the latter. A solution $u$ to (1.1) is constructed such that $u(t, x)$ is $x$-analytic on the time-dependent domain $B_{\rho(t)}\left(\mathrm{I}_{\xi(t)}\right)$ for any $t \in[-T,+T]$. The size parameters $\rho(t), \xi(t)$ decreases as $|t|$ increases. The motivation is that $\partial_{x}$ is a bounded operator between the spaces of analytic functions on $B_{\rho^{\prime}}\left(\mathrm{I}_{\xi^{\prime}}\right)$ and on $B_{\rho}\left(\mathrm{I}_{\xi}\right)$, respectively, for any $\rho<\rho^{\prime}$ and $\xi \leq \xi^{\prime}$. The norm of $\partial_{x}$ is determined by the classical Cauchy estimate

$$
\sup _{x \in B_{\rho}\left(\mathrm{I}_{\xi}\right)}\left|\partial_{x} u(x)\right| \leq\left(\rho^{\prime}-\rho\right)^{-1} \sup _{x \in B_{\rho^{\prime}}\left(\mathrm{I}_{\xi^{\prime}}\right)}|u(x)| .
$$

Introducing suitable time-dependent norms $\|\cdot\|_{t}$ for $u(t)$, an a priori estimate of Gronwall type is derived from (3.1),

$$
\|u(t)\|_{t} \leq\left\|u_{0}\right\|_{0}+\int_{0}^{t} C(t, s)\|u(s)\|_{s} d s .
$$

Eventually, (3.2) enables one to obtain the solution $u$ as the fixed point of a contracting map.

Time-dependent norms for discrete functions are introduced in the following. To prove approximation of $u$ by $v^{\varepsilon}$, a discrete analogue of (3.2) is derived for the $t$-norm of the difference $w^{\varepsilon}(t):=u(t)-v^{\varepsilon}(t)$. More precisely, $w^{\varepsilon}(t)$ at time $t=n \frac{\varepsilon}{2}$ is estimated in terms of $w^{\varepsilon}\left(t-\frac{\varepsilon}{2}\right)$ and $w^{\varepsilon}(t-\varepsilon)$, leading to the discrete Gronwall estimate in (3.16). The crucial technical tool is a discrete version of the Cauchy estimate (3.1), which is given in Lemma 3.1 below.

3.1. Notation. Let $D(\rho) \subset \mathbb{C}$ denote the complex disc of radius $\rho$ centered at 0 , and for $p>1, D^{p}(\rho)=D(\rho) \times \cdots \times D(\rho) \subset \mathbb{C}^{p}$ is a $p$-dimensional poly-disc. Also, recall that $\mathrm{I}_{\xi}=[-\xi,+\xi]$ and $B_{\rho}\left(\mathrm{I}_{\xi}\right)=\left\{z \in \mathbb{C} \mid \operatorname{dist}\left(z, \mathrm{I}_{\xi}\right) \leq \rho\right\}$. 
The crucial quantity that determines most of the following constructions is $r>0$, the appropriate diameter of the domain $\Omega(r)$, which has to be found. From $r$, one defines

$$
\xi(t)=r-|t|, \xi_{n}=r-|n| \frac{\varepsilon}{2}, \rho(t)=3 r-2|t|, \rho_{n}=3 r-|n| \varepsilon .
$$

Discrete intervals containing $m+1$ points are given by

$$
\mathcal{J}_{m}^{\varepsilon}=\left\{\begin{array}{cc}
\left\{-\frac{m}{2} \varepsilon, \ldots,-\varepsilon, 0, \varepsilon, \ldots, \frac{m}{2} \varepsilon\right\} & \text { if } m \text { is even } \\
\left\{-\frac{m}{2} \varepsilon, \ldots,-\frac{\varepsilon}{2}, \frac{\varepsilon}{2}, \ldots, \frac{m}{2} \varepsilon\right\} & \text { if } m \text { is odd }
\end{array}\right.
$$

For a function $v: \mathcal{J}_{m}^{\varepsilon} \rightarrow \mathbb{C}^{d}$, define its difference quotient, $\frac{\varepsilon}{2}$-shift, restriction and linear interpolation:

$$
\begin{array}{rlrl}
\delta_{x} v: \mathcal{J}_{m-1}^{\varepsilon} & \rightarrow \mathbb{C}^{d}, & & \left(\delta_{x} v\right)(x)=\frac{1}{\varepsilon}\left(v\left(x+\frac{\varepsilon}{2}\right)-v\left(x-\frac{\varepsilon}{2}\right)\right), \\
\tau_{x} v: \mathcal{J}_{m-1}^{\varepsilon} \rightarrow \mathbb{C}^{d}, & & \left(\tau_{x} v\right)(x)=v\left(x+\frac{\varepsilon}{2}\right),\left(\tau_{x}^{-1} v\right)(x)=v\left(x-\frac{\varepsilon}{2}\right), \\
\pi v: \mathcal{J}_{m-2}^{\varepsilon} \rightarrow \mathbb{C}^{d}, & & (\pi v)(x)=v(x), \\
E v: \mathrm{I}_{\frac{\varepsilon}{2} m} \rightarrow \mathbb{C}^{d}, & (E v)(x)=\frac{x-x_{-}^{\varepsilon}}{\varepsilon} v\left(x_{+}^{\varepsilon}\right)+\frac{x_{+}^{\varepsilon}-x}{\varepsilon} v\left(x_{-}^{\varepsilon}\right) .
\end{array}
$$

Here $x_{-}^{\varepsilon}, x_{+}^{\varepsilon} \in \mathcal{J}_{m}^{\varepsilon}$ are such that $x_{-}^{\varepsilon} \leq x<x_{+}^{\varepsilon}$ and $x_{+}^{\varepsilon}=x_{-}^{\varepsilon}+\varepsilon$. The restriction of a function $u: \Omega(r) \rightarrow \mathbb{C}^{d}$ to the discrete domain $\Omega^{\varepsilon}(r)$ is denoted by $[u]^{\varepsilon}$. For the discrete function $v: \Omega^{\varepsilon}(r) \rightarrow \mathbb{C}^{d}$, let $v_{n}$ be its restriction to time $t=n \frac{\varepsilon}{2}$, so $v_{n}(x)=v\left(n \frac{\varepsilon}{2}, x\right)$, which is defined on the interval $\mathcal{J}_{n^{\prime}}^{\varepsilon}$, where $n^{\prime}$ is the largest integer with $\frac{\varepsilon}{2} n^{\prime} \leq \xi_{n}$.

In these notations, the problem (2.1) takes the convenient form $(n>0)$ :

$$
\begin{aligned}
v_{n+1}^{\varepsilon} & =\pi v_{n-1}^{\varepsilon}+\varepsilon \delta_{x} v_{n}^{\varepsilon}+\varepsilon f_{n}^{\varepsilon}\left(v^{\varepsilon}\right), \\
f_{n}^{\varepsilon}\left(v^{\varepsilon}\right) & =F^{\varepsilon}\left(\tau_{x} v_{n}^{\varepsilon}, \tau_{x}^{-1} v_{n}^{\varepsilon}, \pi v_{n-1}^{\varepsilon}\right),
\end{aligned}
$$

where pointwise evaluation of the arguments is understood.

Without loss of generality, assume that $|M u| \leq|u|$ for all $u \in \mathbb{C}^{d}$, and that $u_{0}(0)=0$. Indeed, this can be achieved by a dilation of the $t$-axis and an affine transformation of the values of $u$, respectively. For an appropriate choice of $U>0$, $f$ is defined on $D^{d}(3 U)$ and $F^{\varepsilon}$ on $D^{3 d}(3 U)$, respectively.

3.2. Discrete norms and their properties. For $\rho>0$, define a functional on scalar discrete functions $v: \mathcal{J}_{m}^{\varepsilon} \rightarrow \mathbb{C}$ by

$$
\|v\|_{\rho}=\sum_{k=0}^{n} \frac{\rho^{k}}{k !} \max _{x \in \mathcal{J}_{m-k}^{\varepsilon}}\left|\delta^{k} v(x)\right|
$$

For multi-component functions $v=\left(v_{(1)}, \ldots, v_{(p)}\right): \mathcal{J}_{m}^{\varepsilon} \rightarrow \mathbb{C}^{p}$, let

$$
\|v\|_{\rho}=\max _{i=1, \ldots, p}\left\|v_{(i)}\right\|_{\rho}
$$

These norms share several properties with the maximum-norm of analytic functions over a complex domain. 
Lemma 3.1. The functionals $\|\cdot\|_{\rho}$ provide a scale of norms on discrete functions. For $u: \mathcal{J}_{n}^{\varepsilon} \rightarrow \mathbb{C}^{d}$ and $0 \leq \rho \leq \rho^{\prime}$, one has $\|u\|_{\rho} \leq\|u\|_{\rho^{\prime}}$. In addition, each $\|\cdot\|_{\rho}$ has the following properties:

(1) Absolute bound: $|u(x)| \leq\|u\|_{\rho}$ for all $x \in \mathcal{J}_{n}^{\varepsilon}$.

(2) Submultiplicativity: $\|u v\|_{\rho} \leq\|u\|_{\rho}\|v\|_{\rho}$ for scalar functions $u$ and $v$.

(3) Discrete Cauchy estimate: For $\theta>0,\|u\|_{\rho}+\theta\left\|\delta_{x} u\right\|_{\rho} \leq\|u\|_{\rho+\theta}$.

(4) Restriction estimate: If $u: \mathrm{I}_{\xi} \rightarrow \mathbb{C}^{d}$ extends analytically to $B_{\rho^{\prime}}\left(\mathrm{I}_{\xi}\right)$ and $u^{\varepsilon}$ is its restriction to $\mathcal{J}_{n}^{\varepsilon} \subset \mathrm{I}_{\xi}$, then, provided $\rho<\rho^{\prime}$,

$$
\left\|u^{\varepsilon}\right\|_{\rho} \leq\left(1-\rho / \rho^{\prime}\right)^{-1} \sup _{B_{\rho^{\prime}}\left(\mathrm{I}_{\xi}\right)}|u| .
$$

(5) Analyticity estimate: Let functions $v^{\varepsilon}: \mathcal{J}_{n^{\varepsilon}}^{\varepsilon} \rightarrow \mathbb{C}^{d}$ be given, with $n^{\varepsilon} \varepsilon \geq$ $\xi \geq 0$ and $\left\|v^{\varepsilon}\right\|_{\rho} \leq C$ for a sequence $\varepsilon \rightarrow 0$. Then there exists an analytic $u: \mathrm{I}_{\xi} \rightarrow \mathbb{C}^{d}$, so that $E \delta_{x}^{k} v^{\varepsilon(k)}$ converges to $\partial_{x}^{k} u$ uniformly for each $k \geq 0$ and a suitable subsequence $\varepsilon(k) \rightarrow 0$ of $\varepsilon$. Moreover, u possesses a complex extension to $B_{\rho}\left(\mathrm{I}_{\xi}\right)$ which is bounded by $C$.

This lemma is proven in the appendix.

A norm with properties (11) and (2) will be called a submultiplicative norm in the following. For an arbitrary submultiplicative norm, the following lemma holds:

Lemma 3.2. Let the analytic function $f: D^{p}(U) \rightarrow \mathbb{C}$ satisfy

$$
|f(u)| \leq \mathcal{C}\left(\left|u_{(1)}\right|, \ldots,\left|u_{(p)}\right|\right)
$$

for all $u \in D^{p}(U)$, with some function $\mathcal{C} \geq 0$ that is nondecreasing in each of its arguments. Then for each $\gamma>1$ and every discrete function $v: \mathcal{J}_{n}^{\varepsilon} \rightarrow \mathbb{C}^{p}$ with $\gamma\|v\|_{\rho} \leq U$, the composition $f(v)$ is well defined on $\mathcal{J}_{n}^{\varepsilon}$, and

$$
\|f(v)\|_{\rho} \leq \Gamma \mathcal{C}\left(\gamma\left\|v_{(1)}\right\|_{\rho}, \ldots, \gamma\left\|v_{(p)}\right\|_{\rho}\right) .
$$

The constant $\Gamma$ depends on $\gamma$ but not on the submultiplicative norm $\|\cdot\|_{\rho}$.

This lemma is proven in the appendix. Two cases of particular interest are:

$$
\begin{aligned}
\|f(v)\|_{\rho} & \leq \Gamma \sup _{D^{p}(U)}|f|, \\
\left\|f\left(v_{1}\right)-f\left(v_{2}\right)\right\|_{\rho} & \leq \Gamma \sup _{D^{p}(U)}\left|f^{\prime}\right|\left\|v_{1}-v_{2}\right\|_{\rho},
\end{aligned}
$$

which hold for all $v, v_{1}, v_{2}$ with $\|v\|_{\rho} \leq U / \gamma$ and $\left\|v_{1}\right\|_{\rho},\left\|v_{2}\right\|_{\rho} \leq U /(2 \gamma)$, respectively, with $\gamma>1$ arbitrary and $\Gamma=\Gamma(\gamma)$.

Estimate (3.7) follows immediately with $\mathcal{C} \equiv \sup _{D^{p}(U)}|f|$ in (3.6). To obtain (3.8), consider the function $\tilde{f}(a, b):=f(a+b, a-b)$ which is analytic in $a, b \in$ $D^{p}(U / 2)$, and let $\mathcal{C}(|a|,|b|):=\sup _{D^{p}(U)}\left|f^{\prime}\right||b|$. Now let $a=\left(v_{1}+v_{2}\right) / 2$ and $b=$ $\left(v_{1}-v_{2}\right) / 2$.

3.3. Existence of a continuous solution. In the following, it will be shown that for an appropriate $r>0$ and $\varepsilon$ small enough, discrete solutions $v^{\varepsilon}$ exist on $\Omega^{\varepsilon}(r)$, and a limiting function $u$ can be defined on $\Omega(r)$. In fact, $r>0$ will be defined so that the solutions $v^{\varepsilon}$ on $\Omega^{\varepsilon}(r)$ satisfy

$$
\left\|v_{n}^{\varepsilon}\right\|_{\sigma_{n}} \leq U, \quad \sigma_{n}=\rho_{n}+r=4 r-n \varepsilon
$$


for all $n$ with $\frac{\varepsilon}{2}|n| \leq r$; recall that $v_{n}^{\varepsilon}(x)=v^{\varepsilon}\left(n \frac{\varepsilon}{2}, x\right)$. Choose $U>0$ suitable and let $\mathcal{F}>0$ be an $\varepsilon$-independent bound for $F^{\varepsilon}$ on $D^{3 d}(3 U)$. The basic idea is to derive (3.9) inductively as follows:

$$
\mathcal{M}_{n}^{\varepsilon}:=\left\|v_{n+1}^{\varepsilon}\right\|_{\sigma_{n}}+\left\|v_{n}^{\varepsilon}\right\|_{\sigma_{n}} \leq U\left(\frac{1}{2}+\frac{n}{2 N}\right) .
$$

To obtain (3.10) at $n=0$, observe that the functions

$$
u_{0} \text { and } u_{0}+\frac{\varepsilon}{2}\left(M \partial_{x} u_{0}+f\left(u_{0}\right)\right)
$$

are analytic on a complex neighborhood of $x=0$. It follows that if $r$ and $\varepsilon$ are small enough, then the initial data (2.3) satisfies

$$
\left\|v_{0}^{\varepsilon}\right\|_{4 r},\left\|v_{+}^{\varepsilon}\right\|_{4 r} \leq \frac{U}{4}
$$

taking into account that $u_{0}(0)=0$ and using property (4) of Lemma 3.1 .

Now suppose (3.10) holds at $n \geq 0$. Then $f_{n}^{\varepsilon}\left(v^{\varepsilon}\right)$ is defined on $\mathcal{J}_{n}^{\varepsilon}$, and

$$
\begin{aligned}
\mathcal{M}_{n+1} & \leq \underbrace{\left\|v_{n}^{\varepsilon}\right\|_{\sigma_{n+1}}+\varepsilon\left\|\delta_{x} v_{n}^{\varepsilon}\right\|_{\sigma_{n+1}}}_{\leq\left\|v_{n-1}^{\varepsilon}\right\|_{\sigma_{n+1}+\varepsilon}}+\varepsilon \underbrace{\left\|f_{n}^{\varepsilon}\left(v^{\varepsilon}\right)\right\|_{\sigma_{n+1}}}_{\leq \Gamma \mathcal{F}}+\left\|v_{n}^{\varepsilon}\right\|_{\sigma_{n+1}} \\
& \leq \mathcal{M}_{n}+\varepsilon \Gamma \mathcal{F} .
\end{aligned}
$$

To estimate $\delta_{x} v^{\varepsilon}$ and $f^{\varepsilon}\left(v^{\varepsilon}\right)$, property (3) of Lemma 3.1 and inequality (3.7) were employed, respectively.

In addition to $\left|v^{\varepsilon}\right| \leq U$, estimates on the difference quotients follow:

$$
\begin{aligned}
\left|\delta_{x} v^{\varepsilon}\right| & \leq \max _{n}\left(\sigma_{n}^{-1}\left\|v_{n}^{\varepsilon}\right\|_{\sigma_{n}}\right) \leq U /(2 r), \\
\left|\delta_{t} v^{\varepsilon}\right| & \leq\left|\delta_{x} v^{\varepsilon}\right|+\left|f^{\varepsilon}\left(v^{\varepsilon}\right)\right| \leq U / r+\Gamma \mathcal{F}, \\
\left|\delta_{x}^{2} v^{\varepsilon}\right| & \leq \max _{n}\left(2 \sigma_{n}^{-2}\left\|v_{n}^{\varepsilon}\right\|_{\sigma_{n}}\right) \leq U /\left(2 r^{2}\right) .
\end{aligned}
$$

For each $\varepsilon$, let $v_{I}^{\varepsilon}$ be the restriction of $v^{\varepsilon}$ to points $(x, t) \in \Omega^{\varepsilon}(r)$ with "even" time coordinate, $t=(2 k) \frac{\varepsilon}{2}$. Note that $v_{I}^{\varepsilon}$ is defined on a sublattice of $(\varepsilon \mathbb{Z})^{2}$. Define the family $\left\{\hat{v}_{I}^{\varepsilon}\right\}_{\varepsilon}$ of continuous functions obtained from $x$-t-linear interpolation of $v_{I}^{\varepsilon}$ on $\Omega(r)$. By the estimates (3.11) and (3.12), this family is equicontinuous. Hence, the Arzelà-Ascoli theorem applies: There is a sequence $\varepsilon^{\prime} \rightarrow 0$ such that $\hat{v}_{I}^{\varepsilon^{\prime}}$ converges to a smooth limit $u_{I}$ uniformly on $\Omega(r)$. Moreover, by (3.13), it is possible to choose the sequence $\varepsilon^{\prime}$ such that $\delta_{x} \hat{v}_{I}^{\varepsilon^{\prime}}$ also converges uniformly to $\partial_{x} u_{I}$ (cf. the proof of property (5) in Lemma 3.1). The same procedure is applicable to $v_{I I}^{\varepsilon}$, the restriction to the "odd" time values $t=(2 k+1) \frac{\varepsilon}{2}$; choose a subsequence $\varepsilon^{\prime \prime}$ of $\varepsilon^{\prime}$ such that $\hat{v}_{I I}^{\varepsilon^{\prime \prime}} \rightarrow u_{I I}$ and $\delta_{x} \hat{v}_{I I}^{\varepsilon^{\prime \prime}} \rightarrow \partial_{x} u_{I I}$. Also, $f_{n}^{\varepsilon}\left(\hat{v}_{I}^{\varepsilon^{\prime \prime}}\right)$ with even $n$ converges to $f\left(u_{I}\right)$ uniformly as can be deduced from the estimate (2.2) - and respectively for $n$ odd, $f_{n}^{\varepsilon}\left(\hat{v}_{I I}^{\varepsilon^{\prime \prime}}\right) \rightarrow f\left(u_{I I}\right)$.

Since $v^{\varepsilon}$ solves (3.3) , it follows for all $(t, x),\left(t^{\prime}, x\right) \in \Omega(r)$,

$$
v_{I}^{\varepsilon^{\prime \prime}}\left(t^{\prime}, x\right)=v_{I}^{\varepsilon^{\prime \prime}}(t, x)+\int_{t}^{t^{\prime}}\left(\delta_{x} v_{I I}^{\varepsilon^{\prime \prime}}(s, x)+f^{\varepsilon^{\prime \prime}}\left(v_{I I}^{\varepsilon^{\prime \prime}}\right)(s, x)\right) d s+\mathcal{O}\left(\varepsilon^{\prime \prime}\right) .
$$

Passing to the uniform limit as $\varepsilon^{\prime \prime} \rightarrow 0$ :

$$
u_{I}\left(t^{\prime}, x\right)=u_{I}(t, x)+\int_{t}^{t^{\prime}}\left(\partial_{x} u_{I I}(s, x)+f\left(u_{I I}\right)(s, x)\right) d s .
$$

The roles of $u_{I}$ and $u_{I I}$ can be interchanged. Consequently, both functions are differentiable in time and satisfy

$$
\partial_{t}\left(\begin{array}{c}
u_{I} \\
u_{I I}
\end{array}\right)=\left(\begin{array}{cc}
0 & M \\
M & 0
\end{array}\right) \partial_{x}\left(\begin{array}{c}
u_{I} \\
u_{I I}
\end{array}\right)+\left(\begin{array}{c}
f\left(u_{I I}\right) \\
f\left(u_{I}\right)
\end{array}\right) .
$$


By property (5) of Lemma 3.1 the estimates $\left\|v_{n}^{\varepsilon}\right\|_{\sigma_{n}} \leq U$ imply for fixed $t \in[-r, r]$ that $u_{I / I I}(t, x)$ extend $x$-analytically to $B_{r+\rho(t)}\left(\mathrm{I}_{\xi(t)}\right)$, where they are bounded by $U$. And since $u_{I / I I}$ solve the equation (3.14), their analytic continuations do as well. So $u_{I}$ and $u_{I I}$ are smooth with respect to $t$, as any $t$-derivative can be expressed in terms of $x$-derivatives and compositions with the analytic function $f$.

The pair $\left(u_{I}, u_{I I}\right)$ is the only solution to (3.14) of that smoothness. This is seen as follows: In the proof of estimate (2.4) below, $u$ could be any $x$-analytic and $t$-smooth solution to the problem (1.1); no reference to the above construction is made. In particular, estimate (2.4) applies to $\left(u_{I}, u_{I I}\right)$ in place of $u$ and the system (3.14) in place of equation (1.1), respectively. But only one smooth function can satisfy (2.4) for all $\varepsilon>0$.

For symmetry reasons, the unique solution $\left(u_{I}, u_{I I}\right)$ to 3.14 with initial conditions $u_{I}(0, x)=u_{I I}(0, x)=u_{0}(x)$ satisfies $u_{I}=u_{I I}=: u$ on $\Omega(r)$. Hence, $u$ solves (1.1) with $u(0, x)=u_{0}(x)$ for $x \in \mathrm{I}_{r}$.

3.4. Approximation in $C^{0}$. For shortness, let $u^{\varepsilon}=[u]^{\varepsilon}$ be the restriction of the smooth solution, and let $w^{\varepsilon}=v^{\varepsilon}-u^{\varepsilon}$ denote the deviation of $v^{\varepsilon}$ from $u$. The idea of the proof is to calculate $\varepsilon$-independent bounds on the expression

$$
\mathcal{L}_{n}:=\left\|w_{n+1}^{\varepsilon}\right\|_{\rho_{n}}+\left\|w_{n}^{\varepsilon}\right\|_{\rho_{n}}
$$

which is defined for $\frac{\varepsilon}{2}|n| \leq r$. Again, only $n \geq 0$ is considered here, and the treatment of $n \leq 0$ is left to the reader. It will be shown that

$$
\mathcal{L}_{n} \leq\left(1+\varepsilon B^{*}\right) \mathcal{L}_{n-1}+C^{*} \varepsilon^{3} \text { and } \mathcal{L}_{0} \leq D^{*} \varepsilon^{2},
$$

leading by the standard Gronwall estimate to

$$
\mathcal{L}_{n} \leq\left(C^{*}+D^{*}\right) e^{r B^{*}} \varepsilon^{2}
$$

which implies (2.4) for $m \geq 0$ and $n=0$.

To prove the estimate (3.16) at an instant of time $t=\varepsilon n$ with $n \geq 1$, express $w_{n+1}^{\varepsilon}$ in terms of $u^{\varepsilon}$ and $v^{\varepsilon}$ at previous time steps:

$$
\begin{array}{ll}
\text { (A) } \mathcal{L}_{n+1} \leq & \left\|\pi w_{n-1}^{\varepsilon}+\delta_{x} w_{n}^{\varepsilon}\right\|_{\rho_{n}}+\left\|w_{n}^{\varepsilon}\right\|_{\rho_{n}} \\
\text { (B) } & +\varepsilon\left\|f_{n}^{\varepsilon}\left(v^{\varepsilon}\right)-f_{n}^{\varepsilon}\left(u^{\varepsilon}\right)\right\|_{\rho_{n}} \\
\text { (C) } & \\
& +\left\|\varepsilon\left(M \delta_{x} u_{n}^{\varepsilon}+f_{n}^{\varepsilon}\left(u^{\varepsilon}\right)\right)-\left(\delta_{t} u^{\varepsilon}\right)_{n}\right\|_{\rho_{n}}
\end{array}
$$

The three resulting expressions $(\mathrm{A})-(\mathrm{C})$ as well as the initial conditions

$$
\text { (IC) } \quad \mathcal{L}_{0}=\left\|w_{0}^{\varepsilon}\right\|_{\rho_{0}}+\left\|w_{1}^{\varepsilon}\right\|_{\rho_{0}}
$$

are estimated separately in the following.

(A) By property (3), and observing that $\rho_{n}+\varepsilon=\rho_{n-1}$,

$$
\begin{aligned}
(\mathrm{A}) & \leq\left\|w_{n}^{\varepsilon}\right\|_{\rho_{n}}+\varepsilon\left\|\delta_{x} w_{n}^{\varepsilon}\right\|_{\rho_{n}}+\left\|w_{n-1}^{\varepsilon}\right\|_{\rho_{n}} \\
& \leq\left\|w_{n}^{\varepsilon}\right\|_{\rho_{n}+\varepsilon}+\left\|w_{n-1}^{\varepsilon}\right\|_{\rho_{n}} \\
& \leq \mathcal{L}_{n-1} .
\end{aligned}
$$


(B) With the help of estimate (3.5), one concludes

$$
\begin{aligned}
& \left\|f^{\varepsilon}\left(v^{\varepsilon}\right)-f^{\varepsilon}\left(u^{\varepsilon}\right)\right\|_{\rho_{n}} \\
& \quad \leq\left\|F^{\varepsilon}\left(\tau_{x} v_{n}^{\varepsilon}, \tau_{x}^{-1} v_{n}^{\varepsilon}, v_{n-1}^{\varepsilon}\right)-F^{\varepsilon}\left(\tau_{x} u_{n}^{\varepsilon}, \tau_{x}^{-1} u_{n}^{\varepsilon}, u_{n-1}^{\varepsilon}\right)\right\|_{\rho_{n}} \\
& \quad \leq \Gamma \sup \left|D F^{\varepsilon}\right| \max \left(\left\|v_{n}^{\varepsilon}-u_{n}^{\varepsilon}\right\|_{\rho_{n}},\left\|v_{n-1}^{\varepsilon}-u_{n-1}^{\varepsilon}\right\|_{\rho_{n}}\right) \\
& \quad \leq B^{*} \mathcal{L}_{n-1} .
\end{aligned}
$$

The constant $B^{*}$ formally depends on $\varepsilon$ via $\sup _{D^{3 d}(U)}\left|D F^{\varepsilon}\right|$. But the analytic function $F^{\varepsilon}$ is uniformly bounded on $D^{p}(U)$ independently of $\varepsilon$ because of (2.2), and so are its derivatives. Hence $(\mathrm{B}) \leq \varepsilon B^{*} \mathcal{L}_{n-1}$.

(C) Define the functions

$$
\begin{aligned}
& A_{x}^{\varepsilon}(t, x)=\partial_{x} u(t, x)-\frac{1}{\varepsilon}\left(u\left(t, x+\frac{\varepsilon}{2}\right)-u\left(t, x-\frac{\varepsilon}{2}\right)\right) \\
& A_{t}^{\varepsilon}(t, x)=\partial_{t} u(t, x)-\frac{1}{\varepsilon}\left(u\left(t+\frac{\varepsilon}{2}, x\right)-u\left(t-\frac{\varepsilon}{2}, x\right)\right) \\
& A_{f}^{\varepsilon}(t, x)=f(u(x, t))-F^{\varepsilon}\left(u\left(t, x+\frac{\varepsilon}{2}\right), u\left(t, x-\frac{\varepsilon}{2}\right), u\left(x-\frac{\varepsilon}{2}, t\right)\right) .
\end{aligned}
$$

For $t \in[-r,+r]$, all three functions possess an analytic extension for $x \in B_{\rho(t)+r}\left(\mathrm{I}_{\xi(t)}\right)$. As $u$ is smooth, one trivially has

$$
\left|A_{x}^{\varepsilon}(x, t)\right| \leq C_{x} \varepsilon^{2} \quad \text { and } \quad\left|A_{t}^{\varepsilon}(x, t)\right| \leq C_{t} \varepsilon^{2} .
$$

Furthermore, because of estimate (2.2),

$$
\begin{aligned}
\left|A_{f}^{\varepsilon}(x, t)\right| \leq & K\left(\varepsilon+\left|u\left(t, x+\frac{\varepsilon}{2}\right)-u\left(t, x-\frac{\varepsilon}{2}\right)\right|\right)^{2} \\
& +\sup |D f|\left|\left(u\left(t, x+\frac{\varepsilon}{2}\right)+u\left(t, x-\frac{\varepsilon}{2}\right)\right) / 2-u(t, x)\right| \\
\leq & C_{f} \varepsilon^{2} .
\end{aligned}
$$

Subtracting $0=M \partial_{x} u+f(u)-\partial_{t} u$ from the expression for $(\mathrm{C})$,

$$
\begin{aligned}
(\mathrm{C}) & =\left\|M \delta_{x}[u]_{n}^{\varepsilon}+f_{n}^{\varepsilon}\left([u]^{\varepsilon}\right)-\delta_{t}[u]_{n}^{\varepsilon}-\left[M \partial_{x} u+f(u)-\partial_{t} u\right]_{n}^{\varepsilon}\right\|_{\rho_{n}} \\
& \leq\left\|\delta_{x}[u]_{n}^{\varepsilon}-\left[\partial_{x} u\right]_{n}^{\varepsilon}\right\|_{\rho_{n}}+\left\|\delta_{t}[u]_{n}^{\varepsilon}-\left[\partial_{t} u\right]_{n}^{\varepsilon}\right\|_{\rho_{n}}+\left\|f_{n}^{\varepsilon}\left([u]^{\varepsilon}\right)-[f(u)]_{n}^{\varepsilon}\right\|_{\rho_{n}} \\
& \leq\left\|\left[A_{x}^{\varepsilon}\right]_{n}^{\varepsilon}\right\|_{\rho_{n}}+\left\|\left[A_{t}^{\varepsilon}\right]_{n}^{\varepsilon}\right\|_{\rho_{n}}+\left\|\left[A_{f}^{\varepsilon}\right]_{n}^{\varepsilon}\right\|_{\rho_{n}} \\
& \leq \Gamma\left(C_{x}+C_{t}+C_{f}\right) \varepsilon^{2}=C^{*} \varepsilon^{2} .
\end{aligned}
$$

For the conclusive estimate, property (4) of Lemma 3.1 has been used.

(IC) Obviously, $w_{0}^{\varepsilon}=0$ by (2.3). And $v_{1}^{\varepsilon}=v_{+}^{\varepsilon}$ is the restriction of $u_{+}^{\varepsilon}:=$ $u_{0}+\frac{\varepsilon}{2} \partial_{t} u_{0}$, which is an $x$-analytic function on $B_{4 r-\varepsilon}\left(\mathrm{I}_{r-\frac{\varepsilon}{2}}\right)$ and satisfies

$$
\left|u_{+}^{\varepsilon}(x)-u\left(\frac{\varepsilon}{2}, x\right)\right| \leq \frac{\varepsilon^{2}}{8} \sup _{0<s<\varepsilon / 2}\left|\partial_{t}^{2} u(s, x)\right| .
$$

Exploiting the estimate (3.5) yields

$$
\mathcal{L}_{0} \leq\left\|w_{1}^{\varepsilon}\right\|_{r-\varepsilon} \leq D^{*} \varepsilon^{2}
$$

3.5. Proof of smooth approximation. Since $u$ is smooth on $\Omega(r)$, derivatives and difference quotients may be interchanged at the cost of $\mathcal{O}\left(\varepsilon^{2}\right)$ :

$$
\sup _{\Omega_{m+n}^{\varepsilon}(r)}\left|\partial_{x}^{m} \partial_{t}^{n} u-\delta_{x}^{m} \delta_{t}^{n}[u]^{\varepsilon}\right| \leq C_{m n}^{(1)} \varepsilon^{2} .
$$

This is a consequence of the more general formula (5.19) given in Lemma 5.5 in section 5 . Hence, to prove (2.4) for given $m, n$, it suffices to show that

$$
\left|\delta_{x}^{m} \delta_{t}^{n} w^{\varepsilon}(x, t)\right| \leq C_{m n}^{(2)} \varepsilon^{2} .
$$


Submultiplicative norms for functions $v: \Omega^{\varepsilon}(r) \rightarrow \mathbb{R}^{d}$ are given by

$$
\|v\|_{\theta}^{(N)}=\sum_{m=1,2, \ldots} \frac{\theta^{m+n}}{m ! n !} \sup _{(x, t) \in \Omega_{m+n}^{\varepsilon}(r)}\left|\delta_{x}^{m} \delta_{t}^{n} v(x, t)\right| .
$$

Define positive numbers $\theta_{N}=\theta_{0} /(N+1)$, where $\theta_{0}<r$ is suitably chosen later. It is inductively shown that

$$
\left\|w^{\varepsilon}\right\|_{\theta_{N}}^{(N)} \leq C_{N}^{(3)} \varepsilon^{2}
$$

with appropriate constants $C_{N}^{(3)}$. Obviously, (3.23) implies (3.22) with the choice $C_{m n}^{(2)}=C_{n}^{(3)} m ! n ! \theta_{n}^{-(m+n)}$.

Firstly, some properties of the norms are summarized. In contrast to the previously considered norms $\|\cdot\|_{\rho}$, which are defined for restrictions of $v$ to intervals $\mathfrak{J}_{n}^{\varepsilon}$, the $\|\cdot\|_{\theta}^{(N)}$ above involve the whole time-dependent function $v$ at once. Apart from that, each $\|\cdot\|_{\theta}^{(N)}$ constitutes a submultiplicative norm, so Lemma 3.2 applies, and (3.7), (3.8) hold. Also, the discrete Cauchy estimate (property (3) of Lemma 3.1) carries over. Furthermore, the restriction estimate (property (4)) reads as follows: If $\tilde{u}$ is a smooth function such that $\tilde{u}(t)$ is $x$-analytic on $B_{\rho}(\mathrm{I})$ for all $t \in[-r,+r]$, and $0<\theta<\rho$, then

$$
\left\|[\tilde{u}]^{\varepsilon}\right\|_{\theta}^{(N)} \leq \Gamma \max _{n \leq N} \sup _{|t| \leq r} \sup _{x \in D_{\rho}(\mathrm{I})}\left|\partial_{t}^{n} \tilde{u}(t, x)\right|,
$$

where $\Gamma$ depends on the ratio $\theta / \rho$ only.

For $N=0$, inequality (3.23) follows from the previous discussion,

$$
\left\|w^{\varepsilon}\right\|_{\theta_{0}}^{(0)} \leq \max _{n}\left\|w_{n}^{\varepsilon}\right\|_{\rho_{n}}=\mathcal{O}\left(\varepsilon^{2}\right)
$$

because $\theta_{0}<r \leq \rho_{n}$. Now assume (3.23) for $N \geq 0$. By definition of $\|\cdot\|_{\theta}^{(N)}$, and since $v^{\varepsilon}$ solves the discrete equation (2.1),

$$
\begin{aligned}
& \left\|w^{\varepsilon}\right\|_{\theta_{N+1}}^{(N+1)} \leq\left\|w^{\varepsilon}\right\|_{\theta_{N+1}}^{(N)}+\frac{\theta_{N+1}}{N+1}\left\|\delta_{t} w^{\varepsilon}\right\|_{\theta_{N+1}}^{(N)} \\
& \quad \leq\left\|w^{\varepsilon}\right\|_{\theta_{N+1}}^{(N)}+\frac{\theta_{N+1}}{N+1}\left\|\delta_{x} w^{\varepsilon}\right\|_{\theta_{N+1}}^{(N)}+\frac{\theta_{0}}{N^{2}}\left\|f^{\varepsilon}\left(u^{\varepsilon}\right)-f^{\varepsilon}\left(v^{\varepsilon}\right)\right\|_{\theta_{N}}^{(N)}+\frac{\theta_{0}}{N^{2}}\left\|\Delta^{\varepsilon}\right\|_{\theta_{N}}^{(N)} .
\end{aligned}
$$

The Cauchy estimate is applied to the sum of the first two terms, and inequality (3.8) to the third term. The norm of

$$
\Delta^{\varepsilon}=M \delta_{x}[u]^{\varepsilon}+f^{\varepsilon}\left([u]^{\varepsilon}\right)-\delta_{t}[u]^{\varepsilon}=\left[A_{x}^{\varepsilon}\right]^{\varepsilon}+\left[A_{t}^{\varepsilon}\right]^{\varepsilon}+\left[A_{f}^{\varepsilon}\right]^{\varepsilon}
$$

with $A_{x}^{\varepsilon}, A_{t}^{\varepsilon}$ and $A_{f}^{\varepsilon}$ defined as in (3.18) (3.20), is estimated using (3.24),

$$
\left\|\Delta^{\varepsilon}\right\|_{\theta_{N}}^{(N)} \leq \Gamma \max _{n \leq N} \sup _{x, t}\left(\left|\partial_{t}^{n} A_{x}^{\varepsilon}\right|+\left|\partial_{t}^{n} A_{t}^{\varepsilon}\right|+\left|\partial_{t}^{n} A_{f}^{\varepsilon}\right|\right) \leq C_{N}^{(4)} \varepsilon^{2} .
$$

In conclusion,

$$
\begin{aligned}
\left\|w^{\varepsilon}\right\|_{\theta_{N+1}}^{(N+1)} & \leq\left\|w^{\varepsilon}\right\|_{\theta_{N}}^{(N)}+\frac{\theta_{0} \Gamma}{N^{2}} \sup |D f|\left\|w^{\varepsilon}\right\|_{\theta_{N}}^{(N)}+\frac{\theta_{0}}{N^{2}} C_{N}^{(4)} \varepsilon^{2} \\
& \leq\left(\left(1+\frac{\theta_{0} \Gamma}{N^{2}} \sup |D f|\right) C_{N}^{(3)}+C_{N}^{(4)}\right) \varepsilon^{2} .
\end{aligned}
$$

As inequality (3.8) has been applied to estimate $f^{\varepsilon}\left(u^{\varepsilon}\right)-f^{\varepsilon}\left(v^{\varepsilon}\right)$, it needs to be checked that $\left\|u^{\varepsilon}\right\|_{\theta_{N}}^{(N)},\left\|v^{\varepsilon}\right\|_{\theta_{N}}^{(N)} \leq U$. To verify the bound for $v^{\varepsilon}$, formally estimate 
along the same lines as above

$$
\begin{aligned}
\left\|v^{\varepsilon}\right\|_{\theta_{N+1}}^{(N+1)} & \leq\left\|v^{\varepsilon}\right\|_{\theta_{N+1}}^{(N)}+\frac{\theta_{N+1}}{N+1}\left\|\delta_{x} v^{\varepsilon}\right\|_{\theta_{N+1}}^{(N)}+\frac{\theta_{0}}{N^{2}}\left\|f^{\varepsilon}\left(v^{\varepsilon}\right)\right\|_{\theta_{N+1}}^{(N)} \\
& \leq\left\|v^{\varepsilon}\right\|_{\theta_{N}}^{(N)}+\frac{\theta_{0}}{N^{2}} \Gamma \mathcal{F} \leq\left\|v^{\varepsilon}\right\|_{\theta_{0}}^{(0)}+\theta_{0} \Gamma \mathcal{F} \sum_{n \leq N} 1 / n^{2} .
\end{aligned}
$$

For simplicity, assume that $\left\|v^{\varepsilon}\right\|_{\theta_{0}}^{(0)}<\frac{1}{2} U$, possibly after diminishing $r$. As the sum $\sum_{n=0}^{\infty} 1 / n^{2}$ is finite, it can be achieved that $\left\|v^{\varepsilon}\right\|_{\theta_{N}}^{(N)}<\frac{3}{4} U$ for all $N \geq 0$ by choosing $\theta_{0}$ small enough. For $\varepsilon<\varepsilon_{N}$ so small that $Q_{N} \varepsilon^{2}<U / 4$,

$$
\left\|u^{\varepsilon}\right\|_{\theta_{N}}^{(N)} \leq\left\|v^{\varepsilon}\right\|_{\theta_{N}}^{(N)}+\left\|w^{\varepsilon}\right\|_{\theta_{N}}^{(N)} \leq U .
$$

This justifies estimate (3.25) and finishes the proof.

\section{A REMARK ON NUMERICAL APPLICABILITY}

The iteration (3.3) solving the discrete problem (2.1) is tailored to numerical implementation. Assume the values of $v_{n-1}^{\varepsilon}$ and $v_{n}^{\varepsilon}$ are known. Then:

$$
v_{n+1}^{\varepsilon}(x)=v_{n-1}^{\varepsilon}(x)+M\left(v_{n}^{\varepsilon}\left(x+\frac{\varepsilon}{2}\right)-v_{n}^{\varepsilon}\left(x-\frac{\varepsilon}{2}\right)\right)+\varepsilon f_{n}^{\varepsilon}\left(v^{\varepsilon}\right)(x) .
$$

To investigate the effect of round-off errors, consider the perturbed quantity $\hat{v}^{\varepsilon}$ solving the modified equation

$$
\hat{v}_{n}^{\varepsilon}(x)=\hat{v}_{n+2}^{\varepsilon}(x)+M\left(\hat{v}_{n+1}^{\varepsilon}\left(x+\frac{\varepsilon}{2}\right)-\hat{v}_{n+1}^{\varepsilon}\left(x-\frac{\varepsilon}{2}\right)\right)+\varepsilon f_{n}^{\varepsilon}(\hat{v})(x)+\mu_{n}(x) .
$$

Usually, only an absolute bound on the round-off error $\mu$ is known, $\left|\mu_{n}(x)\right| \leq 10^{-q}$. One should think of $q$ as the number of "precise" digits, which is fixed a priori. To estimate the deviation of $\hat{v}^{\varepsilon}$ from $u$, modify inequality (3.16) in the obvious way:

$$
\hat{\mathcal{L}}_{n} \leq\left(1+\varepsilon B^{*}\right) \hat{\mathcal{L}}_{n+1}+C^{*} \varepsilon^{3}+E^{*},
$$

where $E^{*}$ bounds the error introduced by $\mu$. In the worst case, some component of $\mu$ takes values $+10^{-q}$ and $-10^{-q}$ interchangingly, so that

$$
E^{*}=\left\|\mu_{n}\right\|_{\rho_{n}} \approx 10^{-q} \exp (r / \varepsilon) .
$$

As before, $r$ is the diameter of the domain $\Omega$. The relation (4.2) predicts instability of the discrete equation (4.1). A finer mesh size $\varepsilon$ does not necessarily correspond to a better approximation. One expects that there is an $\varepsilon_{0}>0$ (depending on the initial data $u_{0}$ and radius $r>0$ ) such that the approximation error

- decays like $\sim \varepsilon^{2}$ for $\varepsilon \searrow \varepsilon_{0}$,

- grows dramatically like $\sim e^{1 / \varepsilon}$ for $\varepsilon \rightarrow 0$ and $\varepsilon<\varepsilon_{0}$.

From (4.2) it is suggested that $\varepsilon_{0} \sim r / q$. The pictures in the following sections were calculated with $r \approx 1, \varepsilon \approx 0.1$ and $q \approx 10$.

\section{Cross-Ratio equation}

The cross-ratio of four mutually distinct complex numbers $q_{1}, \ldots, q_{4} \in \mathbb{C}$ is

$$
C R\left(q_{1}, q_{2}, q_{3}, q_{4}\right)=\frac{\left(q_{1}-q_{2}\right)\left(q_{3}-q_{4}\right)}{\left(q_{2}-q_{3}\right)\left(q_{4}-q_{1}\right)} .
$$

Consider $\Omega(R)$ as a subset of $\mathbb{C}$, i.e, identify each point $(t, x) \in \Omega(R)$ with the complex number $z=x+i t \in \mathbb{C}$. 

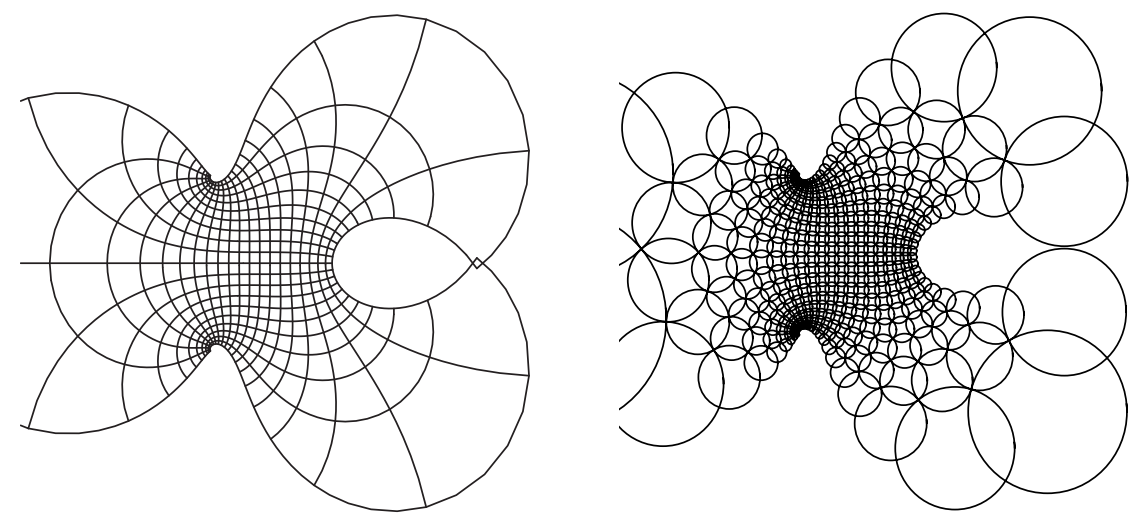

Figure 2. An Airy function is approximated by a CR-mapping.

Definition $5.1(|\mathrm{NQC}|)$. A $C R$-mapping is a lattice-function $\psi^{\varepsilon}: \Omega^{\varepsilon}(R) \rightarrow \mathbb{C}$ such that for all $z_{*} \in \Omega_{*}^{\varepsilon}(R) \subset \mathbb{C}$,

$$
C R\left(\psi^{\varepsilon}\left(z_{*}+\frac{\varepsilon}{2}\right), \psi^{\varepsilon}\left(z_{*}+i \frac{\varepsilon}{2}\right), \psi^{\varepsilon}\left(z_{*}-\frac{\varepsilon}{2}\right), \psi^{\varepsilon}\left(z_{*}-i \frac{\varepsilon}{2}\right)\right)=-1 .
$$

CR-mappings are suitable discrete analogues of holomorphic functions in the sense that any holomorphic function can be locally approximated.

Theorem 5.2. Assume $\phi: \Omega(R) \rightarrow \mathbb{C}$ is a holomorphic function with $\phi^{\prime}(0) \neq 0$. Then there are positive constants $r<R$ and $C_{m n}$, so that for each $\varepsilon>0, a C R$ mapping $\psi^{\varepsilon}$ is defined on $\Omega^{\varepsilon}(r)$ and approximates $\phi$ in $C^{\infty}$ :

$$
\sup _{z \in \Omega_{m+n}^{\varepsilon}(r)}\left|\delta_{x}^{m} \delta_{t}^{n} \psi^{\varepsilon}(z)-\partial_{x}^{m} \partial_{t}^{n} \phi(z)\right| \leq C_{m n} \varepsilon^{2} .
$$

Proof. As a first step, an equation of type (2.1) is derived for an arbitrary CRmapping $\psi^{\varepsilon}: \Omega^{\varepsilon}(R) \rightarrow \mathbb{C}$. Denote by $T_{ \pm}$the shift operators

$$
\left(T_{+} h\right)(z)=h\left(z+\frac{\varepsilon}{2}(i+1)\right), \quad\left(T_{-} h\right)(z)=h\left(z+\frac{\varepsilon}{2}(i-1)\right) .
$$

The edges,

$$
\alpha^{\varepsilon}=\left(T_{+} \psi^{\varepsilon}-\psi^{\varepsilon}\right) / \varepsilon, \quad \beta^{\varepsilon}=\left(T_{-} \psi^{\varepsilon}-\psi^{\varepsilon}\right) / \varepsilon,
$$

naturally satisfy the closure condition

$$
\alpha^{\varepsilon}+T_{+} \beta^{\varepsilon}=\beta^{\varepsilon}+T_{-} \alpha^{\varepsilon} .
$$

Let further the function $Q^{\varepsilon}$ be given as the quotient

$$
Q^{\varepsilon}=\beta^{\varepsilon} / \alpha^{\varepsilon} \text {. }
$$

In terms of these variables, the cross-ratio equation (5.1) implies $T_{+} \beta=-\left(Q^{\varepsilon}\right)^{-1} T_{-} \alpha$, and the following two formulas are obtained:

$$
T_{-} \alpha^{\varepsilon}=\frac{1-Q^{\varepsilon}}{1+Q^{\varepsilon}} Q^{\varepsilon} \alpha^{\varepsilon}, \quad T_{+} \alpha^{\varepsilon}=-\frac{1-Q^{\varepsilon}}{1+Q^{\varepsilon}} \frac{\alpha^{\varepsilon}}{T_{+} Q^{\varepsilon}} .
$$

Their compatibility condition $T_{+}\left(T_{-} \alpha^{\varepsilon}\right)=T_{-}\left(T_{+} \alpha^{\varepsilon}\right)$ provides a discrete analogue of the Cauchy-Riemann equation:

$$
\frac{Q^{\varepsilon}\left(z+i \frac{\varepsilon}{2}\right)}{Q^{\varepsilon}\left(z-i \frac{\varepsilon}{2}\right)}=\frac{1+Q^{\varepsilon}\left(z+\frac{\varepsilon}{2}\right)}{1-Q^{\varepsilon}\left(z+\frac{\varepsilon}{2}\right)} \cdot \frac{1-Q^{\varepsilon}\left(z-\frac{\varepsilon}{2}\right)}{1+Q^{\varepsilon}\left(z-\frac{\varepsilon}{2}\right)} \quad \text { for all } z \in \Omega_{*}^{\varepsilon}(R)
$$


Now introduce $v^{\varepsilon}: \Omega_{*}^{\varepsilon}(R) \rightarrow \mathbb{C}$ by

$$
i \exp \left(\varepsilon v^{\varepsilon}(z)\right)=Q^{\varepsilon}\left(z-i \frac{\varepsilon}{2}\right)
$$

Expressing the relation (5.7) in terms of $v^{\varepsilon}$, one obtains

$$
\delta_{t} v^{\varepsilon}=i \delta_{x} v^{\varepsilon}+F^{\varepsilon}\left(v^{\varepsilon}\right)
$$

which is in the form of the discrete equation (2.1) with $M=i$ and

$$
\begin{aligned}
F^{\varepsilon}\left(v^{+}, v^{-}, v^{*}\right) & =\frac{1}{\varepsilon^{2}}\left(g\left(\varepsilon v^{+}\right)-g\left(\varepsilon v^{-}\right)\right), \\
g(V) & =\log \left(-i \exp (V) \frac{1+i \exp (V)}{1-i \exp (V)}\right) .
\end{aligned}
$$

Note that $v^{\varepsilon}$ is defined on $\Omega_{*}^{\varepsilon}(R)$ instead of $\Omega^{\varepsilon}(R)$. Except for a minor mix-up of notation, this obviously does not affect any of the results.

Lemma 5.3. For any solution $v^{\varepsilon}: \Omega_{*}^{\varepsilon}(r) \rightarrow \mathbb{C}$ to (5.9), there exists a corresponding CR-mapping $\psi^{\varepsilon}: \Omega^{\varepsilon}(r) \rightarrow \mathbb{C}$. It is uniquely determined by $v^{\varepsilon}$ up to Euclidean motions and a homothety.

Proof of Lemma 5.3, Let $\hat{z}$ be the "bottom" of $\Omega^{\varepsilon}(r)$, i.e., $\hat{z}=-i r^{\varepsilon} \in \Omega^{\varepsilon}(r)$ with $r-\frac{\varepsilon}{2}<r^{\varepsilon} \leq r$. Assign $\alpha^{\varepsilon}(\hat{z})$ an arbitrary, nonzero number $A^{\varepsilon} \in \mathbb{C}$. Equation (5.8) defines $Q^{\varepsilon}$ from $v^{\varepsilon}$, and (5.6) defines $\alpha^{\varepsilon}$ on $\Omega^{\varepsilon}(r)$. Consistency is provided, i.e. $T_{+}\left(T_{-} \alpha^{\varepsilon}\right)=T_{-}\left(T_{+} \alpha^{\varepsilon}\right)$, because $Q^{\varepsilon}$ solves (5.7). Let $\beta^{\varepsilon}=Q^{\varepsilon} \alpha^{\varepsilon}$. Assign an arbitrary number $\Psi^{\varepsilon} \in \mathbb{C}$ to $\psi^{\varepsilon}(\hat{z})$, then define $\psi^{\varepsilon}$ on $\Omega^{\varepsilon}(r)$ according to (5.3). Compatibility (5.4) is guaranteed because $\alpha^{\varepsilon}$ solves (5.6). To verify that $\psi^{\varepsilon}$ is indeed a CR-mapping, combine equations (5.6) and (5.4). Variations of $A^{\varepsilon}$ correspond to rigid rotations and dilations of the whole lattice $\psi^{\varepsilon}$, variations of $\Psi^{\varepsilon}$ to translations.

Proof of Theorem 5.2, continued: The continuous counterpart of $v^{\varepsilon}$ is

$$
u(z)=-\frac{1}{2} \frac{\phi^{\prime \prime}(z)}{\phi^{\prime}(z)}
$$

which is defined on some $\Omega(R)$ with $R>0$ small enough (since $\phi^{\prime}(0) \neq 0$ by hypothesis). To justify this ansatz, define

$$
a^{\varepsilon}=\left(T_{+} \phi-\phi\right) / \varepsilon, \quad b^{\varepsilon}=\left(T_{-} \phi-\phi\right) / \varepsilon
$$

as analogues of $\alpha^{\varepsilon}$ and $\beta^{\varepsilon}$, respectively. By Taylor expansion, one finds

$$
\begin{aligned}
b^{\varepsilon}(z) & =i \exp \left(\varepsilon u\left(z+i \frac{\varepsilon}{2}\right)\right) a^{\varepsilon}(z)+\mathcal{O}\left(\varepsilon^{2}\right), \\
T_{-} a^{\varepsilon}(z) & =\frac{1-i \exp \left(\varepsilon u\left(z+i \frac{\varepsilon}{2}\right)\right)}{1+i \exp \left(\varepsilon u\left(z+i \frac{\varepsilon}{2}\right)\right)} i \exp \left(\varepsilon u\left(z+i \frac{\varepsilon}{2}\right)\right) a^{\varepsilon}(z)+\mathcal{O}\left(\varepsilon^{2}\right),
\end{aligned}
$$

corresponding to (5.5) and (5.6), respectively. The Cauchy-Riemann equation for $u$,

$$
\partial_{t} u=i \partial_{x} u,
$$

corresponds to $M=i$ and $f \equiv 0$ in problem (1.1). Since $g(0)=g^{\prime}(0)=g^{\prime \prime}(0)=0$ by (5.11), one easily verifies $\left|F^{\varepsilon}\left(v^{+}, v^{-}, v^{*}\right)\right| \leq K \varepsilon \mid v^{+}-v^{-}$, so the function $F^{\varepsilon}$ in (5.10) satisfies the estimate (2.2). Let $v^{\varepsilon}$ be the solution to (5.9) with initial data (2.3) obtained from the function $u$ in (5.12). Theorem 2.2 yields approximation of $u$ by $v^{\varepsilon}$, according to estimate (2.4). 
By Lemma 5.3. $v^{\varepsilon}$ corresponds to a CR-mapping $\psi^{\varepsilon}$. Fix $\psi^{\varepsilon}$ by choosing $\psi^{\varepsilon}(\hat{z})=$ $\phi(\hat{z})$ and $\alpha^{\varepsilon}(\hat{z})=\left(T_{+} \phi(\hat{z})-\phi(\hat{z})\right) / \varepsilon$. It remains to be shown that approximation of $u$ by $v^{\varepsilon}$ implies approximation of $\phi$ by $\psi^{\varepsilon}$. Smooth approximation is again proven with the help of suitable norms, which are in this case

$$
\left\|w^{\varepsilon}\right\|^{(N)}=\sum_{m+n \leq N} \frac{1}{m ! n !} \max _{z \in \Omega_{m+n}^{\varepsilon}(r)}\left|\delta_{x}^{m} \delta_{t}^{n} w^{\varepsilon}(z)\right| .
$$

This norm is submultiplicative, so Lemma 3.2 applies. The other essential ingredient is

Lemma 5.4. For $v: \Omega^{\varepsilon}(r) \rightarrow \mathbb{C}^{d}$, and $N \geq 0$, one has

$$
\|v\|^{(N+1)} \leq C_{r}\left(\left|v\left(z_{1}\right)\right|+\left|v\left(z_{2}\right)\right|+\left\|\delta_{x} v\right\|^{(N)}+\left\|\delta_{t} v\right\|^{(N)}\right),
$$

where $z_{1}, z_{2} \in \Omega^{\varepsilon}(r)$ are arbitrary points with $0<\left|z_{1}-z_{2}\right|<\varepsilon$.

Proof of Lemma 5.4. From the definition, it is clear that

$$
\|v\|^{(N+1)} \leq \max _{z \in \Omega^{\varepsilon}(r)}|v(z)|+\left\|\delta_{x} v\right\|^{(N)}+\left\|\delta_{t} v\right\|^{(N)} .
$$

Write $z_{1}=\left(t_{1}, x_{1}\right), z_{2}=\left(t_{2}, x_{2}\right)$ and let $z=(t, x) \in \Omega^{\varepsilon}(r)$ be arbitrary. There exist integers $P$ and $Q$ with $t=t_{i}+\varepsilon P$ and $x=x_{i}+\varepsilon Q$ for either $i=1$ or $i=2$. Naturally, $\varepsilon|P|+\varepsilon|Q| \leq 2 r$. Thus

$$
\begin{aligned}
|v(z)| & \leq\left|v\left(z_{i}\right)\right|+\varepsilon \sum_{p=1}^{P}\left|\delta_{x} v\left(z_{i}+p^{\prime} \varepsilon-\frac{\varepsilon}{2}\right)\right|+\varepsilon \sum_{q=1}^{Q}\left|\delta_{t} v\left(z-i q \varepsilon+i \frac{\varepsilon}{2}\right)\right| \\
& \leq\left|v\left(z_{i}\right)\right|+2 r\left(\left\|\delta_{x} v\right\|^{(N)}+\left\|\delta_{t} v\right\|^{(N)}\right) .
\end{aligned}
$$

So (5.18) holds with $C_{r}=1+2 r$.

Proof of Theorem 5.2, continued: At this point, it is sensible to introduce the notion $\tilde{u}^{\varepsilon}=\mathcal{O}_{\infty}\left(\varepsilon^{p}\right)$ for a family of smooth functions $\tilde{u}^{\varepsilon}: \Omega(r) \rightarrow \mathbb{C}^{d}$, meaning that $\left\|\tilde{u}^{\varepsilon}\right\|_{C^{N}(\Omega)}<C_{N} \varepsilon^{p}$ for all $N \geq 0$, with suitable constants $C_{N}>0$.

Lemma 5.5. Let $\tilde{u}: \Omega(r) \rightarrow \mathbb{C}^{d}$ be an arbitrary smooth function. For natural numbers $m, n$, one has

$$
\left[\partial_{x}^{m} \partial_{t}^{n} \tilde{u}\right]^{\varepsilon}=\delta_{x}^{m} \delta_{t}^{n}[\tilde{u}]^{\varepsilon}+\mathcal{O}_{\infty}\left(\varepsilon^{2}\right) .
$$

Moreover, for $\lambda_{x}, \lambda_{t} \in \mathbb{R}$, define $\tilde{u}_{ \pm}^{\varepsilon}(x, t)=\tilde{u}\left(x \pm \varepsilon \lambda_{x}, t \pm \varepsilon \lambda_{t}\right)$. Then

$$
\begin{aligned}
& \tilde{u}_{+}^{\varepsilon}+\tilde{u}_{-}^{\varepsilon}=2 \tilde{u}+\mathcal{O}_{\infty}\left(\varepsilon^{2}\right), \\
& \tilde{u}_{+}^{\varepsilon}-\tilde{u}_{-}^{\varepsilon}=2 \varepsilon\left(\lambda_{x} \partial_{x} \tilde{u}+\lambda_{t} \partial_{t} \tilde{u}\right)+\mathcal{O}_{\infty}\left(\varepsilon^{3}\right) .
\end{aligned}
$$

The identity (5.19) is proven in the appendix. The derivation of the properties (5.20), (5.21) is analogous.

It is clear from Theorem 2.2 and Lemma 5.5 that $\left\|[u]^{\varepsilon}-v^{\varepsilon}\right\|^{(N)}=\mathcal{O}\left(\varepsilon^{2}\right)$ for arbitrary $N$. It is now shown that this implies

$$
\left\|\alpha^{\varepsilon}-a^{\varepsilon}\right\|^{(N)}=\mathcal{O}\left(\varepsilon^{2}\right) .
$$

Introduce the functions $g^{\varepsilon}, G^{\varepsilon}$ by

$$
\alpha(z)=\exp \left(g^{\varepsilon}(z)\right) \alpha(\hat{z}), \quad a(z)=\exp \left(G^{\varepsilon}(z)\right) a(\hat{z}) .
$$


Combining the formulas in (5.6) yields the simple relation

$$
\tau_{x} \alpha^{\varepsilon}=T_{-}^{-1}\left(T_{+} \alpha^{\varepsilon}\right)=\frac{-1}{\left(\tau_{x} Q^{\varepsilon}\right)\left(T_{-}^{-1} Q^{\varepsilon}\right)} \alpha^{\varepsilon} \text {, so } \delta_{x} g^{\varepsilon}=-\left(T_{+} v^{\varepsilon}+v^{\varepsilon}\right) .
$$

Adopting the notion $\mathcal{O}_{\infty}\left(\varepsilon^{2}\right)$, and using the rules (5.20) and (5.21),

$$
\begin{aligned}
\partial_{x} G^{\varepsilon}(z)= & \frac{T_{+} \phi^{\prime}-\phi^{\prime}}{T_{+} \phi-\phi}(z)=\frac{\phi^{\prime \prime}}{\phi^{\prime}}\left(z+\frac{1+i}{2} \frac{\varepsilon}{2}\right)+\mathcal{O}_{\infty}\left(\varepsilon^{2}\right) \\
& =-2 u\left(z+\frac{1+i}{2} \frac{\varepsilon}{2}\right)+\mathcal{O}_{\infty}\left(\varepsilon^{2}\right)=-\left(T_{+} u+u\right)(z)+\mathcal{O}_{\infty}\left(\varepsilon^{2}\right) .
\end{aligned}
$$

Similar identities are derived for $\delta_{t} g^{\varepsilon}$ and $\partial_{t} G^{\varepsilon}$, so that

$$
\left\|\delta_{x}\left(g^{\varepsilon}-G^{\varepsilon}\right)\right\|^{(N)}+\left\|\delta_{t}\left(g^{\varepsilon}-G^{\varepsilon}\right)\right\|^{(N)} \leq 4\left\|[u]^{\varepsilon}-v^{\varepsilon}\right\|^{(N)}+\mathcal{O}\left(\varepsilon^{2}\right)=\mathcal{O}\left(\varepsilon^{2}\right) .
$$

As $\alpha^{\varepsilon}(\hat{z})=a^{\varepsilon}(\hat{z})$ by construction, the asymptotic estimates (5.14), (5.15) yield $T_{-} \alpha^{\varepsilon}(\hat{z})=T_{-} a^{\varepsilon}(\hat{z})+\mathcal{O}\left(\varepsilon^{2}\right)$. Hence also $g^{\varepsilon}(\hat{z})=G^{\varepsilon}(\hat{z})=0$ and $T_{-} g^{\varepsilon}(\hat{z})=$ $T_{-} G^{\varepsilon}(\hat{z})+\mathcal{O}\left(\varepsilon^{2}\right)$. Lemma 5.4 gives

$$
\left\|G^{\varepsilon}-g^{\varepsilon}\right\|^{(N)} \leq C_{N}\left\|[u]^{\varepsilon}-v^{\varepsilon}\right\|^{(N)} .
$$

By means of the composition estimate (3.8),

$$
\left\|\alpha^{\varepsilon}-a^{\varepsilon}\right\|^{(N)} \leq\left\|\exp \left(g^{\varepsilon}\right)-\exp \left(G^{\varepsilon}\right)\right\|^{(N)}|\alpha(\hat{z})|=\mathcal{O}\left(\varepsilon^{2}\right),
$$

and this implies (5.22). An analogous argument gives the respective approximation of $b^{\varepsilon}$ by $\beta^{\varepsilon}$.

In the second step, $\psi^{\varepsilon}$ and $\phi$ are reconstructed from $\alpha^{\varepsilon}, \beta^{\varepsilon}$ and $a^{\varepsilon}, b^{\varepsilon}$, respectively. By definition of these quantities,

$$
\begin{array}{ll}
\delta_{x} \psi^{\varepsilon}(z)=\alpha^{\varepsilon}\left(z-\frac{\varepsilon}{2}\right)-\beta^{\varepsilon}\left(z+\frac{\varepsilon}{2}\right), & \delta_{x}[\phi]^{\varepsilon}(z)=a^{\varepsilon}\left(z-\frac{\varepsilon}{2}\right)-b^{\varepsilon}\left(z+\frac{\varepsilon}{2}\right), \\
\delta_{t} \psi^{\varepsilon}(z)=\alpha^{\varepsilon}\left(z-i \frac{\varepsilon}{2}\right)+\beta^{\varepsilon}\left(z+\frac{\varepsilon}{2}\right), & \delta_{t}[\phi]^{\varepsilon}(z)=a^{\varepsilon}\left(z-i \frac{\varepsilon}{2}\right)+b^{\varepsilon}\left(z+\frac{\varepsilon}{2}\right) .
\end{array}
$$

By construction of $\psi^{\varepsilon}$, one has $\psi^{\varepsilon}(\hat{z})=\phi(\hat{z})$, and also $T_{+} \psi^{\varepsilon}(\hat{z})=T_{+} \phi(\hat{z})+$ $\mathcal{O}\left(\varepsilon^{2}\right)$ because $\alpha^{\varepsilon}(\hat{z})=a^{\varepsilon}(\hat{z})+\mathcal{O}\left(\varepsilon^{2}\right)$. Applying Lemma 5.4 to $[\phi]^{\varepsilon}$ and $\psi^{\varepsilon}$ gives $\left\|[\phi]^{\varepsilon}-\psi^{\varepsilon}\right\|^{(N)}=\mathcal{O}\left(\varepsilon^{2}\right)$ and thus the desired final result (5.2).

\section{Schramm's orthogonal Patterns}

In [Sch], Schramm-patterns (or orthogonal circle patterns) are proposed as discrete analogues of conformal maps. A Schramm-pattern $\mathcal{C}^{\varepsilon}$ assigns to each vertex $(x, t) \in \tilde{\Omega}^{\varepsilon} \subset(\varepsilon \mathbb{Z})^{2}$ a circle $\mathcal{C}^{\varepsilon}(x, t)$ in $\mathbb{R}^{2} \equiv \mathbb{C}$. The defining condition is that circles belonging to neighboring vertices intersect orthogonally, and circles assigned to opposite corners of an $(\varepsilon \mathbb{Z})^{2}$-square are tangent. So $\mathcal{C}^{\varepsilon}(x, t)$ and $\mathcal{C}^{\varepsilon}\left(x^{\prime}, t^{\prime}\right)$ intersect orthogonally and are tangent, respectively, if $\left|x-x^{\prime}\right|+\left|t-t^{\prime}\right|=\varepsilon$ and if $\left|x-x^{\prime}\right|=\left|t-t^{\prime}\right|=\varepsilon$. For the formal definition, refer to the original article.

The theory developed here allows for an easy proof of local $C^{\infty}$-approximation of conformal maps. The obtained local result differs in nature from the $C^{0}$-convergence theorem presented in $[\mathbf{S c h}$, which deals with global boundary value problems.

For notational simplicity, the domain for an orthogonal circle pattern $\mathfrak{C}^{\varepsilon}$ is

$$
\tilde{\Omega}^{\varepsilon}(r)=\Omega^{\varepsilon}(r) \cap(\varepsilon \mathbb{Z})^{2},
$$

containing half of the grid points of $\Omega^{\varepsilon}(r)$. In obvious analogy to $\Omega_{k}^{\varepsilon}(r)$, the sets $\tilde{\Omega}_{m n}^{\varepsilon}(r) \subset \Omega_{m+n}^{\varepsilon}(r)$ are introduced so that the central difference quotient $\delta_{x}^{m} \delta_{t}^{n} \psi^{\varepsilon}$ of a function $\psi^{\varepsilon}$ on $\tilde{\Omega}^{\varepsilon}(r)$ is naturally evaluated on $\tilde{\Omega}_{m n}^{\varepsilon}(r)$. Note that $\tilde{\Omega}_{m n}^{\varepsilon}$ and $\tilde{\Omega}_{n m}^{\varepsilon}$ do not coincide in general. 

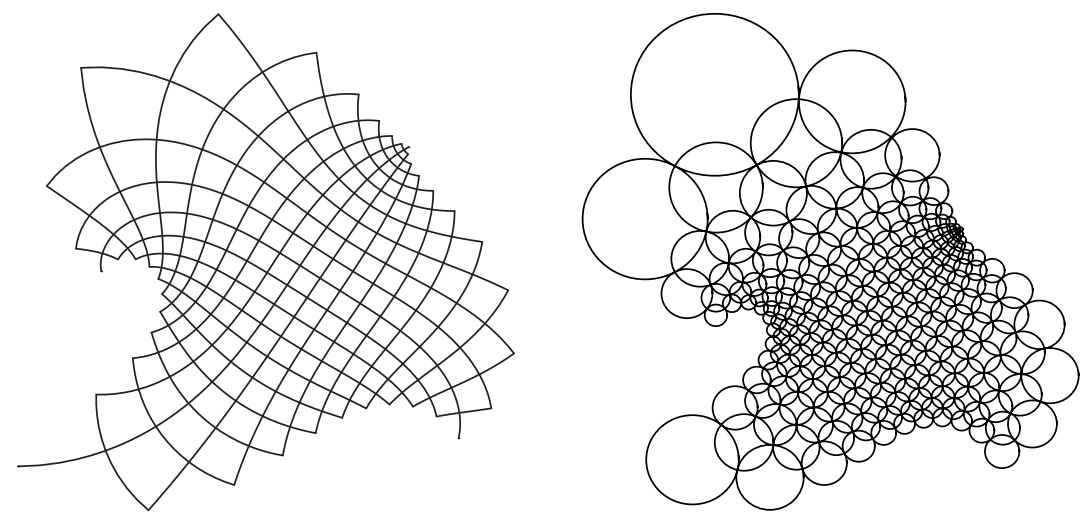

Figure 3. An Airy function is approximated by a Schramm-pattern.

By the results in $\mathrm{Sch}$, a pattern $\mathcal{C}^{\varepsilon}$ is - up to rigid motions - determined by its radius function $\rho^{\varepsilon}$, which assigns to each point $(t, x) \in(\varepsilon \mathbb{Z})^{2}$ the radius of the circle $\mathcal{C}^{\varepsilon}(t, x)$.

Theorem 6.1. Given a conformal map $\phi: \Omega(R) \rightarrow \mathbb{C}$ with $\partial_{x} \phi(0) \neq 0$, there is a positive $r<R$, and there exists a family of orthogonal circle patterns $\mathcal{C}^{\varepsilon}$ defined on $\tilde{\Omega}^{\varepsilon}(r)$ for all $\varepsilon>0$ small enough, whose radii functions $\rho^{\varepsilon}$ are convergent to the metric factor $\rho=\left|\partial_{x} \phi\right|$ of $\phi$ in $C^{\infty}$,

$$
\sup _{(x, t) \in \tilde{\Omega}_{m n}^{\varepsilon}(r)}\left|\delta_{x}^{m} \delta_{t}^{n} \rho^{\varepsilon}(x, t)-\partial_{x}^{m} \partial_{t}^{n} \rho(x, t)\right| \leq C_{m n} \varepsilon^{2} .
$$

Proof. The function $\log \rho$ is harmonic, i.e., satisfies Laplace's equation

$$
\partial_{x}^{2}(\log \rho)+\partial_{t}^{2}(\log \rho)=0 .
$$

In terms of $u_{(1)}=\partial_{x}(\log \rho)$ and $u_{(2)}=\partial_{t}(\log \rho)$, harmonicity reads

$$
\partial_{t}\left(\begin{array}{l}
u_{(1)} \\
u_{(2)}
\end{array}\right)=\left(\begin{array}{cc}
0 & 1 \\
-1 & 0
\end{array}\right) \partial_{x}\left(\begin{array}{l}
u_{(1)} \\
u_{(2)}
\end{array}\right) .
$$

For the radius function $\rho^{\varepsilon}$ of a Schramm-pattern, an "exponential Laplace equation" has been derived in $[\mathrm{Sch}]$. In our notations,

$$
\frac{\left(\tau_{x}^{2} \rho^{\varepsilon}\right)\left(\tau_{t}^{2} \rho^{\varepsilon}\right)\left(\tau_{x}^{-2} \rho^{\varepsilon}\right)\left(\tau_{t}^{-2} \rho^{\varepsilon}\right)}{\left(\rho^{\varepsilon}\right)^{2}}=\frac{\left(\tau_{x}^{2} \rho^{\varepsilon}\right)+\left(\tau_{t}^{2} \rho^{\varepsilon}\right)+\left(\tau_{x}^{-2} \rho^{\varepsilon}\right)+\left(\tau_{t}^{-2} \rho^{\varepsilon}\right)}{\left(\tau_{x}^{2} \rho^{\varepsilon}\right)^{-1}+\left(\tau_{t}^{2} \rho^{\varepsilon}\right)^{-1}+\left(\tau_{x}^{-2} \rho^{\varepsilon}\right)^{-1}+\left(\tau_{t}^{-2} \rho^{\varepsilon}\right)^{-1}} .
$$

Recall that $\tau_{x}$ and $\tau_{t}$ denote the $\frac{\varepsilon}{2}$-shift in the $x$ - and $t$-direction, respectively, so that $\tau_{x}^{ \pm 2}$ and $\tau_{t}^{ \pm 2}$ are shifts by $\varepsilon$. Equation (6.4) is satisfied by a positive function $\rho^{\varepsilon}: \tilde{\Omega}^{\varepsilon}(r) \rightarrow \mathbb{R}_{+}$if and only if it is the radius function of a Schramm-pattern.

Introduce functions $v_{(1)}^{\varepsilon}, v_{(2)}^{\varepsilon}$ by

$$
\exp \left(\varepsilon v_{(1)}^{\varepsilon}\right)=\left(\tau_{x} \rho^{\varepsilon}\right) /\left(\tau_{x}^{-1} \rho^{\varepsilon}\right), \quad \exp \left(\varepsilon v_{(2)}^{\varepsilon}\right)=\left(\tau_{t} \rho^{\varepsilon}\right) /\left(\tau_{t}^{-1} \rho^{\varepsilon}\right) .
$$

Since $\rho^{\varepsilon}$ is given on $\tilde{\Omega}^{\varepsilon}(r), v_{(1)}^{\varepsilon}$ and $v_{(2)}^{\varepsilon}$ are a priori defined on different subsets of $\Omega_{*}^{\varepsilon}(r)$. However, the domains of the difference quotients $\delta_{t} v_{(1)}^{\varepsilon}$ and $\delta_{x} v_{(2)}^{\varepsilon}$ coincide, and the same is true for $\delta_{x} v_{(1)}^{\varepsilon}$ and $\delta_{t} v_{(2)}^{\varepsilon}$. Equation (6.4) and the compatibility 
condition $\delta_{x} v_{(2)}^{\varepsilon}=\delta_{t} v_{(1)}^{\varepsilon}$ imply formally

$$
\begin{aligned}
\delta_{t}\left(\begin{array}{c}
v_{(1)}^{\varepsilon} \\
v_{(2)}^{\varepsilon}
\end{array}\right) & =\left(\begin{array}{cc}
0 & 1 \\
-1 & 0
\end{array}\right) \delta_{x}\left(\begin{array}{c}
v_{(1)}^{\varepsilon} \\
v_{(2)}^{\varepsilon}
\end{array}\right)+\left(\begin{array}{c}
0 \\
G^{\varepsilon}
\end{array}\right), \\
\text { with } G^{\varepsilon} & =\frac{1}{\varepsilon^{2}} \log \left(H^{\varepsilon} / H^{-\varepsilon}\right), \\
H^{\varepsilon}\left(v^{+}, v^{-}, v^{*}\right) & =e^{\varepsilon v_{(1)}^{+}}+e^{-\varepsilon v_{(1)}^{-}}+e^{-\varepsilon v_{(2)}^{*}}-e^{\varepsilon\left(v_{(1)}^{+}-v_{(1)}^{-}-v_{(2)}^{*}\right)} .
\end{aligned}
$$

Suppose $v^{\varepsilon}: \Omega_{*}^{\varepsilon}(r) \rightarrow \mathbb{R}^{2}$ is a solution to (6.6), then the equations 6.5 are compatible. From the components $v_{(1)}^{\varepsilon}$ and $v_{(2)}^{\varepsilon}$, a solution $\rho^{\varepsilon}: \tilde{\Omega}^{\varepsilon}(r) \rightarrow \mathbb{R}_{+}$to (6.5) can be constructed and is uniquely determined up to a global scalar factor. (Note that $v_{(1)}^{\varepsilon}$ and $v_{(2)}^{\varepsilon}$ are defined at more points than needed to calculate $\rho^{\varepsilon}$. Actually, any solution $v^{\varepsilon}$ to (6.6) corresponds to two independent Schramm-patterns.) $\rho^{\varepsilon}$ satisfies the exponential Laplace equation (6.4), hence is the radius function of some Schramm-pattern $\mathcal{C}^{\varepsilon}$ on $\tilde{\Omega}^{\varepsilon}(r)$.

$H$ is an analytic function with respect to $v_{+}, v_{-}, v_{*}$ and $\varepsilon$. Keeping the values of the $v$ 's fixed, a simple calculation shows that

$$
\left.\left(\frac{\mathrm{d}}{\mathrm{d} \varepsilon}\right)^{k}\right|_{\varepsilon=0} \log \left(H^{\varepsilon} / H^{-\varepsilon}\right)=\left\{\begin{array}{cl}
0 & \text { for } k=0,1,2, \\
\left(v_{(1)}^{+}-v_{(1)}^{-}\right) \cdot h(v) & \text { for } k=3,
\end{array}\right.
$$

where $h$ is some analytic expression of the $v^{\text {'s. Hence }}\left|G^{\varepsilon}\right| \leq K \varepsilon\left|v_{+}-v_{-}\right|$, implying the estimate (2.2) for $F$. Now let $v^{\varepsilon}$ be the solution to (6.6) with the restrictions of $u$ as initial data in (2.3). Theorem 2.2 applies and yields convergence of $v^{\varepsilon}$ to $u$ on a suitable $\Omega(r)$.

Make the respective solution $\rho^{\varepsilon}$ of 6.5 unique by choosing $\rho^{\varepsilon}(0,0)=\rho(0,0)$. From estimates completely analogous to those used in the proof of Theorem 5.2 (reconstruction of $\alpha^{\varepsilon}$ from $v^{\varepsilon}$ ) one obtains $C^{\infty}$-convergence of $\rho^{\varepsilon}$ to $\rho$.

The radius function $\rho^{\varepsilon}: \tilde{\Omega}^{\varepsilon}(r) \rightarrow \mathbb{R}_{+}$is accompanied by a function $\psi^{\varepsilon}$ : $\tilde{\Omega}^{\varepsilon}(r) \rightarrow \mathbb{C}$, such that $\mathcal{C}^{\varepsilon}(x, t)$ is the circle of radius $\rho^{\varepsilon}(x, t)>0$ around the center $\psi^{\varepsilon}(x, t) \in \mathbb{C}$.

Theorem 6.2. Under the hypotheses of Theorem 6.1 and for every $\varepsilon>0$ small enough, there exists a Schramm-pattern $\mathcal{C}^{\varepsilon}$ on $\tilde{\Omega}^{\varepsilon}(r)$, such that the circle centers $\psi^{\varepsilon}$ converge to the conformal map $\phi$ in $C^{\infty}$ :

$$
\sup _{(x, t) \in \tilde{\Omega}_{m n}^{\varepsilon}(r)}\left|\delta_{x}^{m} \delta_{t}^{n} \psi^{\varepsilon}(x, t)-\partial_{x}^{m} \partial_{t}^{n} \phi(x, t)\right| \leq C_{m n} \varepsilon^{2} .
$$

Proof. As pointed out before, the radius function alone determines the pattern $\mathfrak{C}^{\varepsilon}$ - and hence $\psi^{\varepsilon}$ - up to a rigid motion. Formulas for the reconstruction of $\phi$ and $\psi^{\varepsilon}$ from $\rho$ and $\rho^{\varepsilon}$, respectively, are now derived.

Introduce the real-valued function $\omega$ on $\Omega(R)$ by

$$
\phi^{\prime}=\rho \exp (i \omega)
$$

where $\phi^{\prime}(x, t):=\partial_{x} \phi(x, t)$ is holomorphic with respect to the complex variable $z=x+i t$. The Cauchy-Riemann equations for $\phi^{\prime}$ read

$$
\begin{aligned}
\partial_{x} \omega & =-\partial_{t} \log \rho=-u_{(2)}, \\
\partial_{t} \omega & =\partial_{x} \log \rho=u_{(1)},
\end{aligned}
$$

with $u$ defined as before, $u_{(1)}=\partial_{x}(\log \rho), u_{(2)}=\partial_{t}(\log \rho)$. 

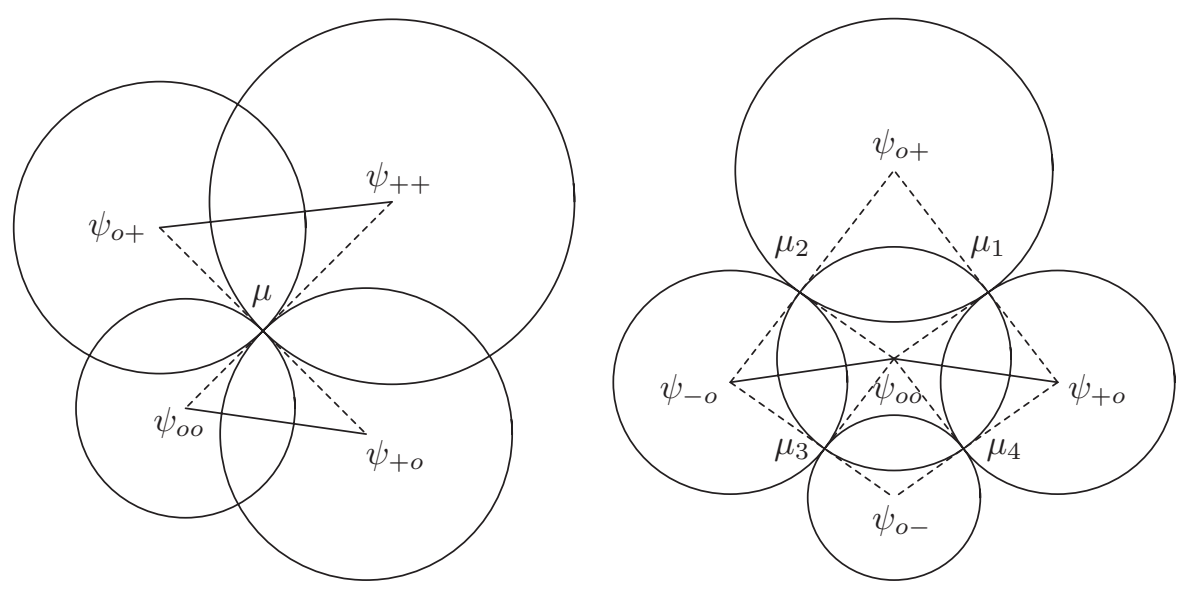

FiguRE 4. Relations between centers of adjacent circles

Analogous quantities and relations are now given for an arbitrary Schrammpattern $\mathcal{C}^{\varepsilon}$. Define the real functions $\omega^{\varepsilon}$ and $d^{\varepsilon}$ on $\tilde{\Omega}_{1,0}^{\varepsilon}(r)$ by

$$
\delta_{x} \psi^{\varepsilon}=d^{\varepsilon} \exp \left(i \omega^{\varepsilon}\right)
$$

with $d^{\varepsilon}(x, t)$ denoting the Euclidian distance between the circle centers $\psi^{\varepsilon}\left(x+\frac{\varepsilon}{2}, t\right)$ and $\psi^{\varepsilon}\left(x+\frac{\varepsilon}{2}, t\right)$, and $\omega^{\varepsilon}(x, t)$ the slope of their connecting line to the $x$-axis.

In Figure 4, two pieces of a Schramm-pattern are displayed. From the left sketch, it is clear that

$$
\begin{aligned}
\angle\left(\psi_{++}-\psi_{0+}, \psi_{+0}-\psi_{00}\right) & =\angle\left(\mu, \psi_{0+}, \psi_{++}\right)-\angle\left(\psi_{00}, \psi_{+0}, \mu\right) \\
& =\arctan \left(\frac{\rho_{++}}{\rho_{0+}}\right)-\arctan \left(\frac{\rho_{00}}{\rho_{+0}}\right) .
\end{aligned}
$$

Introducing $v^{\varepsilon}$ by the formulas 6.5),

$$
\begin{aligned}
\delta_{t} \omega^{\varepsilon} & =g^{\varepsilon}\left(\tau_{t} v_{(1)}^{\varepsilon}, \tau_{t}^{-1} v_{(1)}^{\varepsilon}\right), \\
g^{\varepsilon}\left(v^{+}, v^{-}\right) & =\frac{1}{\varepsilon}\left(\arctan \exp \left(\varepsilon v^{+}\right)-\arctan \exp \left(-\varepsilon v^{-}\right)\right) .
\end{aligned}
$$

From the sketch on the right, it follows that

$$
\begin{aligned}
\angle\left(\psi_{+0}-\psi_{00}, \psi_{00}-\psi_{-0}\right) & =\angle\left(\mu_{4}, \psi_{00}, \mu_{3}\right)-\angle\left(\mu_{1}, \psi_{00}, \mu_{2}\right) \\
& =\arctan \left(\frac{\rho_{0-}}{\rho_{00}}\right)-\arctan \left(\frac{\rho_{0+}}{\rho_{00}}\right) .
\end{aligned}
$$

Expressed in terms of $\omega^{\varepsilon}$ and $v^{\varepsilon}$, this yields:

$$
\delta_{x} \omega^{\varepsilon}=-g^{\varepsilon}\left(\tau_{t} v_{(2)}^{\varepsilon}, \tau_{t}^{-1} v_{(2)}^{\varepsilon}\right) .
$$

With formulas (6.12) and (6.13) at hand, the argument of the proof for Theorem 6.1 is continued. Let $\rho^{\varepsilon}: \tilde{\Omega}^{\varepsilon}(r) \rightarrow \mathbb{R}_{+}$be the radius function approximating the conformal factor $\rho$ of $\phi$, and $v^{\varepsilon}$ the corresponding quantity approximating $u$. Further, let $\psi^{\varepsilon}$ be the function of center positions of the unique Schrammpattern determined by the radius function $\hat{\rho}^{\varepsilon}=\varepsilon \rho^{\varepsilon} / \sqrt{2}$, which also satisfies the side conditions $\psi^{\varepsilon}(0)=\phi(0)$ and $\omega^{\varepsilon}\left(0, \frac{\varepsilon}{2}\right)=\omega\left(0, \frac{\varepsilon}{2}\right)$. 
The same norms (5.17) are used as in the proof of Theorem 5.2 To prove $C^{\infty}$ convergence of $\omega^{\varepsilon}$ to $\omega$, observe that each $g^{\varepsilon}$ is analytic and satisfies

$$
\left|g^{\varepsilon}\left(v^{+}, v^{-}\right)-\left(v^{+}+v^{-}\right) / 2\right| \leq K\left(\varepsilon+\left|v^{+}-v^{-}\right|\right)^{2} .
$$

Restating (6.10) in the form (recall the notion $\mathcal{O}_{\infty}\left(\varepsilon^{2}\right)$ from section 5)

$$
\delta_{t}[w]^{\varepsilon}(x, t)=\frac{1}{2}\left(u\left(x, t+\frac{\varepsilon}{2}\right)+u\left(x, t-\frac{\varepsilon}{2}\right)\right)+\mathcal{O}_{\infty}\left(\varepsilon^{2}\right),
$$

one obtains, using Lemma 3.2 .

$$
\begin{aligned}
\left\|\delta_{t}\left([w]^{\varepsilon}-\omega^{\varepsilon}\right)\right\|^{(N)} & \leq\left\|g^{\varepsilon}\left(\tau_{t} v^{\varepsilon}, \tau_{t}^{-1} v^{\varepsilon}\right)-\frac{1}{2}\left(\tau_{t} v^{\varepsilon}, \tau_{t}^{-1} v^{\varepsilon}\right)\right\|+\left\|\mathcal{O}_{\infty}\left(\varepsilon^{2}\right)\right\|^{(N)} \\
& \leq K\left(\varepsilon+\varepsilon\left\|\delta_{t} v^{\varepsilon}\right\|^{(N)}\right)^{2}+\left\|[u]^{\varepsilon}-v^{\varepsilon}\right\|^{(N)}+\mathcal{O}\left(\varepsilon^{2}\right) .
\end{aligned}
$$

Similarly, $\left\|\delta_{x}\left([\omega]^{\varepsilon}-\omega^{\varepsilon}\right)\right\|^{(N)}=\mathcal{O}\left(\varepsilon^{2}\right)$. Now Lemma 5.4 gives $\left\|[\omega]^{\varepsilon}-\omega^{\varepsilon}\right\|^{(N)}=\mathcal{O}\left(\varepsilon^{2}\right)$. Completely analogous estimates hold for the approximation of $\tilde{\omega}$ and $\tilde{\omega}^{\varepsilon}$ defined by

$$
\begin{aligned}
\partial_{t} \phi & =\rho \exp (i \tilde{\omega}), \\
\delta_{t} \psi^{\varepsilon} & =\tilde{d}^{\varepsilon} \exp \left(i \tilde{\omega}^{\varepsilon}\right) .
\end{aligned}
$$

Equations (6.12) and (6.13) change in the obvious way, and the choice $\omega^{\varepsilon}\left(\frac{\varepsilon}{2}, 0\right)=$ $\omega\left(\frac{\varepsilon}{2}, 0\right)$ implies that $\tilde{\omega}^{\varepsilon}\left(0, \frac{\varepsilon}{2}\right)=\pi / 2+\tilde{\omega}\left(0, \frac{\varepsilon}{2}\right)+\mathcal{O}\left(\varepsilon^{2}\right)$. Another application of Lemma 5.4 yields $\left\|\omega^{\varepsilon}-[\omega]^{\varepsilon}\right\|^{(N)}=\mathcal{O}\left(\varepsilon^{2}\right)$ and $\left\|\tilde{\omega}^{\varepsilon}-[\tilde{\omega}]^{\varepsilon}\right\|^{(N)}=\mathcal{O}\left(\varepsilon^{2}\right)$.

Since the distance between the centers of two orthogonally intersecting circles with radii $\rho_{1}$ and $\rho_{2}$ is

$$
d=\sqrt{\rho_{1}^{2}+\rho_{2}^{2}}=\rho_{1} \sqrt{1+\left(\rho_{2} / \rho_{1}\right)^{2}}
$$

the quantities $d^{\varepsilon}$ and $\tilde{d}^{\varepsilon}$ are given by

$$
d^{\varepsilon}=\sqrt{\frac{1+\exp \left(2 \varepsilon v_{(1)}^{\varepsilon}\right)}{2}}\left(\tau_{x}^{-1} \rho^{\varepsilon}\right), \quad \tilde{d}^{\varepsilon}=\sqrt{\frac{1+\exp \left(2 \varepsilon v_{(2)}^{\varepsilon}\right)}{2}}\left(\tau_{t}^{-1} \rho^{\varepsilon}\right) .
$$

Now observe that 1

$$
\begin{aligned}
\rho & =\sqrt{\rho^{2}}=\sqrt{\frac{\tau_{x}^{-1} \rho^{2}+\tau_{x} \rho^{2}}{2}}+\mathcal{O}_{\infty}\left(\varepsilon^{2}\right)=\sqrt{\frac{1+\left(\tau_{x} \rho / \tau_{x}^{-1} \rho\right)^{2}}{2}}\left(\tau_{x}^{-1} \rho\right)+\mathcal{O}_{\infty}\left(\varepsilon^{2}\right) \\
& =\sqrt{\frac{1+\exp \left(2 \varepsilon u_{(1)}\right)}{2}}\left(\tau_{x}^{-1} \rho\right)+\mathcal{O}_{\infty}\left(\varepsilon^{2}\right) .
\end{aligned}
$$

Hence, with Lemma 3.2 it follows

$$
\begin{aligned}
\left\|\delta_{x}\left(\psi^{\varepsilon}-[\phi]^{\varepsilon}\right)\right\|^{(N)} & \leq C_{N}\left(\left\|\rho^{\varepsilon}-[\rho]^{\varepsilon}\right\|^{(N)}+\left\|\omega^{\varepsilon}-[\omega]^{\varepsilon}\right\|^{(N)}\right)+\mathcal{O}\left(\varepsilon^{2}\right) \\
\left\|\delta_{t}\left(\psi^{\varepsilon}-[\phi]^{\varepsilon}\right)\right\|^{(N)} & \leq C_{N}\left(\left\|\rho^{\varepsilon}-[\rho]^{\varepsilon}\right\|^{(N)}+\left\|\tilde{\omega}^{\varepsilon}-[\tilde{\omega}]^{\varepsilon}\right\|^{(N)}\right)+\mathcal{O}\left(\varepsilon^{2}\right)
\end{aligned}
$$

An application of Lemma 5.4 finishes the argument.

\footnotetext{
${ }^{1}$ The reason formulas (6.16) are employed instead of the symmetric representation $d=$ $\sqrt{\rho_{1}^{2}+\rho_{2}^{2}}$ is that the former is an analytic expression in $\rho, u$ on some $D(U)$ with arbitrarily large $U$ as $\varepsilon \rightarrow 0$, whereas the latter has a singularity at $\rho_{1}=\rho_{2}=0$.
} 


\section{Appendix}

Proof of Lemma 3.1. As a finite sum of norms, $\|\cdot\|_{\rho}$ is seen to constitute a norm itself. The absolute bound is trivial.

Submultiplicativity: For two functions $u, v: \mathcal{J}_{n}^{\varepsilon} \rightarrow \mathbb{C}$,

$$
\begin{aligned}
\|u v\|_{\rho} & =\sum_{k=0}^{n} \frac{\rho^{k}}{k !} \sup _{x \in \mathcal{J}_{n-k}^{\varepsilon}}\left|\delta_{x}^{k}(u v)(x)\right| \\
& \leq \sum_{k \leq n} \frac{\rho^{k}}{k !} \sum_{\ell=0}^{k}\left(\begin{array}{l}
k \\
\ell
\end{array}\right) \sup _{x \in \mathcal{J}_{n-\ell}^{\varepsilon}-\ell}\left|\delta_{x}^{\ell} u(x)\right| \sup _{y \in \mathcal{J}_{n-k+\ell}^{\varepsilon}}\left|\delta_{x}^{k-\ell} v(y)\right| \\
& \leq \sum_{\ell=0}^{n} \sum_{m=0}^{n} \frac{\rho^{\ell+m}}{\ell ! m !} \sup _{x \in \mathcal{J}_{n-\ell}^{\varepsilon}}\left|\delta_{x}^{\ell} u(x)\right| \sup _{y \in \mathcal{J}_{n-m}^{\varepsilon}}\left|\delta_{x}^{m} v(y)\right| \\
& \leq\|u\|_{\rho}\|v\|_{\rho} .
\end{aligned}
$$

Discrete Cauchy estimate: For $u: \mathfrak{J}_{n}^{\varepsilon} \rightarrow \mathbb{C}^{d}$,

$$
\begin{aligned}
\|u\|_{\rho}+\theta\left\|\delta_{x} u\right\|_{\rho} & =\sum_{k=0}^{n} \frac{\rho^{k}}{k !} \max _{x \in \mathcal{J}_{n-k}^{\mathrm{E}}}\left|\delta_{x}^{k} u(x)\right|+\theta \sum_{k=0}^{n-1} \frac{\rho^{k}}{k !} \max _{x \in \mathcal{J}_{n-k-1}^{\varepsilon}}\left|\delta_{x}^{k+1} u(x)\right| \\
& \leq \sum_{k=0}^{n} \frac{\rho^{k}}{k !}\left(1+\theta \frac{k}{\rho}\right) \max _{x \in \mathcal{J}_{n-k}^{\mathcal{E}}}\left|\delta_{x}^{k} u(x)\right| \\
& \leq \sum_{k=0}^{n+1} \frac{(\rho+\theta)^{k}}{k !} \max _{x \in \mathcal{J}_{n-k}^{\varepsilon}}\left|\delta_{x}^{k} u(x)\right|=\|u\|_{\rho+\theta}
\end{aligned}
$$

because for $k=0,1, \ldots$, one has $1+k \theta / \rho \leq(1+\theta / \rho)^{k}$.

Restriction estimate: With the classical Cauchy estimate

$$
\sup _{x \in \mathrm{I}}\left|\partial_{x}^{k} u(x)\right| \leq k !\left(1 / \rho^{\prime}\right)^{k} \sup _{x \in B_{\rho^{\prime}}(\mathrm{I})}|u(x)|
$$

it follows that

$$
\begin{aligned}
\left\|[u]^{\varepsilon}\right\|_{\rho}=\sum_{k=0}^{n} \frac{\rho^{k}}{k !} \sup _{x \in \mathcal{J}_{n-k}^{\varepsilon}}\left|\delta_{x}^{k}[u]^{\varepsilon}(x)\right| & \leq \sum_{k=0}^{n} \frac{\rho^{k}}{k !} \sup _{x \in \mathrm{I}}\left|\partial_{x}^{k} u(x)\right| \\
& \leq\left(\sum_{k=0}^{n}\left(\rho / \rho^{\prime}\right)^{k}\right) \sup _{x \in B_{\rho^{\prime}}(\mathrm{I})}|u(x)| .
\end{aligned}
$$

Analyticity estimate: For simplicity, assume that all $n^{\varepsilon}$ are odd. $\left\|v^{\varepsilon}\right\|_{\rho} \leq C$ implies $\left|\delta^{s} v^{\varepsilon}(x)\right| \leq C s ! \rho^{-s}$ for all $x \in \mathcal{J}_{n^{\varepsilon}}^{\varepsilon}$ and all $s \leq n^{\varepsilon}$. Hence, for fixed $s \geq 0$ and $\varepsilon$ small enough, the sequence of interpolated functions $E \delta_{x}^{s} v^{\varepsilon}$ is equicontinuous.

At $s=0$, the Arzelà-Ascoli theorem yields a subsequence $\varepsilon(0) \rightarrow 0$ of $\varepsilon$ so that $E v^{\varepsilon(0)}$ converges uniformly to a continuous function $u$. From here, proceed inductively: Assume that $E \delta_{x}^{s} v^{\varepsilon(s)}$ converges uniformly to $\partial_{x}^{s} u$. Apply the ArzelàAscoli theorem at $s+1$ to obtain an infinite subsequence $\varepsilon(s+1)$ of $\varepsilon(s)$ for which $E \delta_{x}^{s+1} v^{\varepsilon(s+1)}$ converges uniformly to some $u^{(s+1)}$. To show that $u^{(s+1)}$ is indeed the $s+1$ st derivative of $u$, consider the identity

$$
\left(\delta_{x}^{s} u^{\varepsilon(s+1)}\right)(x)=\left(\delta_{x}^{s} u^{\varepsilon(s+1)}\right)(0)+\varepsilon \sum_{0 \leq j<J}\left(\delta_{x}^{s+1} u^{\varepsilon(s+1)}\right)\left(\frac{\varepsilon}{2}+\varepsilon j\right)
$$


with arbitrary $x=\varepsilon J \in \mathcal{J}_{n^{\varepsilon}-s}^{\varepsilon}$. This implies for the interpolated functions

$$
\left(E \delta_{x}^{s} u^{\varepsilon(s+1)}\right)(x)=\left(E \delta_{x}^{s} u^{\varepsilon(s+1)}\right)(0)+\int_{0}^{x}\left(E \delta_{x}^{s+1} u^{\varepsilon(s+1)}\right)(z) d z+\mathcal{O}\left(\varepsilon^{\prime}\right)
$$

at arbitrary $x \in \mathrm{I}$. Pass to the limit $\varepsilon^{\prime} \rightarrow 0$ on both sides. $u^{(s+1)}$ is seen to be the $x$-derivative of $\partial_{x}^{s} u$, so $u \in C^{s}(\mathrm{I})$. From the theorem on dominated convergence, it follows for $x \in \mathrm{I}$,

$$
\sum_{s=0}^{\infty} \frac{\rho^{s}}{s !}\left|\partial_{x}^{s} u(x)\right| \leq C .
$$

So $u$ possesses a convergent Taylor expansion around all $x \in \mathrm{I}$, with convergence radius $\rho$, and the analytic extension is bounded by $C$.

Proof of Lemma 3.2, As $\left|u_{(i)}(\xi)\right| \leq\|u\|_{\rho}<U / \gamma$ by hypothesis and property (11), the composition $g(u)$ is a well-defined function on the respective $\mathcal{J}_{n}^{\varepsilon}$. The estimate (3.6) is derived as follows: Since $g: D^{p}(U) \rightarrow \mathbb{C}^{d}$ is analytic, it has a power series representation

$$
g(u)=\sum_{\alpha \in \mathbb{N}_{0}^{p}} \frac{\partial^{\alpha} g(0)}{\alpha !} u^{\alpha}, \quad u^{\alpha}=\prod_{j=1}^{p} u_{j}^{\alpha_{j}},
$$

where $\alpha=\left(\alpha_{1}, \ldots, \alpha_{p}\right)$ denotes a multi-index. For the coefficients in (7.1), the Cauchy integral representation yields

$$
\begin{aligned}
\frac{\left|\partial^{\alpha} g(0)\right|}{\alpha !} & =\frac{1}{(2 \pi)^{p}}\left|\int_{\left|\mu_{1}\right|=m_{1}} d \mu_{1} \cdots \int_{\left|\mu_{p}\right|=m_{p}} d \mu_{p} \frac{g\left(\mu_{1}, \ldots, \mu_{p}\right)}{\mu_{1}^{\alpha_{1}+1} \cdots \mu_{p}^{\alpha_{p}+1}}\right| \\
& \leq \frac{F\left(m_{1}, \ldots, m_{p}\right)}{m_{1}^{\alpha_{1}} \cdots m_{p}^{\alpha_{p}}}
\end{aligned}
$$

the $m_{i}$ being arbitrary numbers with $0<m_{i}<U$. Choosing $m_{i}=\gamma\left\|u_{(i)}\right\|_{\rho}$, it follows from submultiplicativity and the basic norm properties of $\|\cdot\|_{\rho}$ that

$$
\begin{aligned}
\|g(u)\|_{\rho} & \leq \sum_{\alpha}\left|\frac{\partial^{\alpha} g(0)}{\alpha !}\right|\left\|u^{\alpha}\right\|_{\rho} \\
& \leq F\left(m_{1}, \ldots, m_{p}\right) \sum_{\alpha}\left(\left(\frac{\left\|u_{(1)}\right\|_{\rho}}{m_{1}}\right)^{\alpha_{1}} \ldots\left(\frac{\left\|u_{(p)}\right\|_{\rho}}{m_{p}}\right)^{\alpha_{p}}\right) \\
& \leq \sum_{\alpha} \gamma^{-|\alpha|} \cdot F\left(\gamma\left\|u_{(1)}\right\|_{\rho}, \ldots, \gamma\left\|u_{(p)}\right\|_{\rho}\right) .
\end{aligned}
$$

This proves the claim and also shows that $\Gamma:=(1-1 / \gamma)^{-p}$ is independent of the norm $\|\cdot\|_{\rho}$.

Proof of Lemma 5.5. One starts with the representation

$$
\delta_{x}^{m} \delta_{t}^{n}[u]^{\varepsilon}(x, t)=\frac{1}{\varepsilon^{m+n}} \int_{\left[-\frac{\varepsilon}{2},+\frac{\varepsilon}{2}\right]^{m}} d^{m} \boldsymbol{\xi} \int_{\left[-\frac{\varepsilon}{2},+\frac{\varepsilon}{2}\right]^{n}} d^{n} \boldsymbol{\tau} \partial_{x}^{m} \partial_{t}^{n} u(x+\bar{\xi}, t+\bar{\tau})
$$

of arbitrary partial difference quotients, where $\boldsymbol{\xi}=\left(\xi_{1}, \ldots, \xi_{m}\right), \boldsymbol{\tau}=\left(\tau_{1}, \ldots, \tau_{n}\right)$, and the notations $\bar{\xi}=\sum_{i=1}^{m} \xi_{i}$ and $\bar{\tau}=\sum_{j=1}^{n} \tau_{j}$ have been used. Then,

$$
\begin{aligned}
& \left(\delta_{x}^{m} \delta_{t}^{n}[u]^{\varepsilon}-\partial_{x}^{m} \partial_{t}^{n} u\right)(x, t)=\frac{\int d \boldsymbol{\xi} d \boldsymbol{\eta}}{\varepsilon^{m+n}} \int_{0}^{1} d s \partial_{(\xi \tau)} \partial_{x}^{m} \partial_{t}^{n} u(x+s \bar{\xi}, t+s \bar{\tau}) \\
& =\frac{\int d \boldsymbol{\xi} d \boldsymbol{\eta}}{\varepsilon^{m+n}} \int_{0}^{1} d s(\underbrace{\partial_{(\xi \tau)} \partial_{x}^{m} \partial_{t}^{n} u(x, t)}_{A(x, t)}+s \int_{0}^{1} d s^{\prime} \underbrace{\partial_{(\xi \tau)}^{2} \partial_{x}^{m} \partial_{t}^{n} u\left(x+s^{\prime} s \bar{\xi}, t+s^{\prime} s \bar{\tau}\right)}_{B(x, t)}) .
\end{aligned}
$$


Above, $\partial_{(\xi \tau)}=(\bar{\xi}) \partial_{x}+(\bar{\tau}) \partial_{t}$. The integral over $A$ vanishes because

$$
\int d^{m} \boldsymbol{\xi} \int d^{n} \boldsymbol{\tau} \partial_{(\xi \tau)} f=0
$$

for an arbitrary $\boldsymbol{\xi}, \boldsymbol{\tau}$-independent function $f$. As $B(x, t)$ is an $(x, t)$-smooth function, one concludes (5.19).

\section{REFERENCES}

[BMS] A.I. Bobenko, D. Matthes, Yu.B. Suris, Discrete and smooth orthogonal systems: $C^{\infty}$ approximation. Int. Math. Res. Not. 45 (2003), 2415-2459. MR2006481

[BP1] A.I. Bobenko, U. Pinkall, Discrete isothermic surfaces, J. Reine Angew. Math. 475 (1996), 187-208. MR1396732 (97f:53004)

[BP2] A.I. Bobenko, U. Pinkall, Discretization of surfaces and integrable systems. in: "Discrete integrable geometry and physics" (Vienna, 1996), Oxford Lecture Ser. Math. Appl., 16, Oxford Univ. Press, New York, 1999. MR1676682 (2001j:37128)

[DHR] P. Doyle, Z.X. He, B. Rodin, Second derivatives of circle packings and conformal mappings. Discrete Comput. Geom. 11 (1994), no. 1, 35-49. MR.1244888 (94m:30017)

[HS] Z.X. He, O. Schramm, The $C^{\infty}$-convergence of hexagonal disk packings to the Riemann map. Acta Math. 180 (1998), no. 2, 219-245. MR1638772 (99j:52021)

[Nag] M. Nagumo, Über das Anfangswertproblem partieller Differentialgleichungen. Jap. J. Math. 18 (1942). 41-47. MR0015186 (7:382e)

[Nir] L. Nirenberg, An abstract form of the nonlinear Cauchy-Kowalewski theorem. J. Differential Geometry 6 (1972), 561-576. MR0322321 (48:683)

[NQC] F.W. Nijhoff, G.R. Quispel, H.W. Capel, Direct linearization of nonlinear differencedifference equations. Phys. Lett. A 97 (1983), no. 4, 125-128. MR0719638 (85j:58078)

[RS] B. Rodin, D. Sullivan, The convergence of circle packings to the Riemann mapping. J. Differential Geom. 26 (1987), no. 2, 349-360. MR0906396 (90c:30007)

[Sch] O. Schramm, Circle patterns with the combinatorics of the square grid. Duke Math. J. 86 (1997), no. 2, 347-389. MR1430437|(98a:30061)

[Wal] W. Walter, An elementary proof of the Cauchy-Kowalevsky theorem. Amer. Math. Monthly 92 (1985), no. 2, 115-126. MR0777557|(87h:35012)

Institut für Mathematik, Technische Universität Berlin, Strasse des 17.Juni 136, 10623 Berlin, Germany

Current address: Institut für Mathematik, Johannes Gutenberg Universität Mainz, Staudingerweg 9, 55128 Mainz, Germany

E-mail address: matthes@mathematik.uni-mainz.de 